\title{
ASYMPTOTICS OF GREEDY ENERGY POINTS
}

\author{
A. LÓPEZ GARCÍA AND E. B. SAFF
}

\begin{abstract}
For a symmetric kernel $k: X \times X \rightarrow \mathbb{R} \cup\{+\infty\}$ on a locally compact metric space $X$, we investigate the asymptotic behavior of greedy $k$-energy points $\left\{a_{i}\right\}_{1}^{\infty}$ for a compact subset $A \subset X$ that are defined inductively by selecting $a_{1} \in A$ arbitrarily and $a_{n+1}$ so that $\sum_{i=1}^{n} k\left(a_{n+1}, a_{i}\right)=$ $\inf _{x \in A} \sum_{i=1}^{n} k\left(x, a_{i}\right)$. We give sufficient conditions under which these points (also known as Leja points) are asymptotically energy minimizing (i.e. have en$\operatorname{ergy} \sum_{i \neq j}^{N} k\left(a_{i}, a_{j}\right)$ as $N \rightarrow \infty$ that is asymptotically the same as $\mathcal{E}(A, N):=$ $\left.\min \left\{\sum_{i \neq j} k\left(x_{i}, x_{j}\right): x_{1}, \ldots, x_{N} \in A\right\}\right)$, and have asymptotic distribution equal to the equilibrium measure for $A$. For the case of Riesz kernels $k_{s}(x, y):=$ $|x-y|^{-s}, s>0$, we show that if $A$ is a rectifiable Jordan arc or closed curve in $\mathbb{R}^{p}$ and $s>1$, then greedy $k_{s}$-energy points are not asymptotically energy minimizing, in contrast to the case $s<1$. (In fact, we show that no sequence of points can be asymptotically energy minimizing for $s>1$.) Additional results are obtained for greedy $k_{s}$-energy points on a sphere, for greedy best-packing points (the case $s=\infty$ ), and for weighted Riesz kernels. For greedy bestpacking points we provide a simple counterexample to a conjecture attributed to L. Bos.
\end{abstract}

\section{INTRODUCTION, BACKGROUND RESULTS AND NOTATION}

The aim of this paper is to study asymptotic properties of special types of extremal point configurations which we shall call greedy energy points. As the name suggests, these configurations are generated by a greedy algorithm which is, in fact, an energy minimizing construction. The notion of energy that we refer to will be specified shortly. We focus on two aspects: the asymptotic behavior of their energy and their limiting distributions, as their cardinality approaches infinity. In many aspects they are similar to minimal (non-greedy) energy configurations, which are those with smallest possible energy. But we will also show that in some situations the behavior of greedy points differs significantly from that of minimal energy points.

Part of the results in this paper are presented in the abstract setting of locally compact metric spaces. Potential theory on more general spaces (such as locally compact Hausdorff spaces) was developed by Choquet [9], 10, Fuglede [17] and

Received by the editor December 12, 2008 and, in revised form, June 27, 2009.

2010 Mathematics Subject Classification. Primary 65D99, 52A40; Secondary 78A30.

Key words and phrases. Minimal energy, Leja points, equilibrium measure, Riesz kernels, bestpacking configurations, Voronoi cells.

The results of this paper form a part of the first author's Ph.D. dissertation at Vanderbilt University.

The research of the second author was supported, in part, by National Science Foundation grants DMS-0603828 and DMS-0808093. 
Ohtsuka [27. Recently Zorii [31, [32] has studied properties of potentials with external fields in this context.

We also investigate greedy configurations in $\mathbb{R}^{p}$, interacting through the so-called Riesz potential $V=1 / r^{s}$, where $s>0$ and $r$ denotes Euclidean distance, as well as greedy "best-packing" points that are chosen to maximize the minimum distance to previously selected points.

Next, we introduce the basic notions necessary to describe our results. We will also present in this section some background material.

Let $X$ denote a locally compact metric space containing infinitely many points. A kernel in $X$ is, by definition, a lower semicontinuous function (l.s.c.) $k: X \times X \rightarrow$ $\mathbb{R} \cup\{+\infty\}$. It is called positive if $k(x, y) \geq 0$ for all $x, y \in X$.

For a set $\omega_{N}=\left\{x_{1}, \ldots, x_{N}\right\}$ of $N(N \geq 2)$ points in $X$, not necessarily distinct, we write $\operatorname{card}\left(\omega_{N}\right)=N$ and define the discrete energy of $\omega_{N}$ by

$$
E\left(\omega_{N}\right):=\sum_{1 \leq i \neq j \leq N} k\left(x_{i}, x_{j}\right)=\sum_{i=1}^{N} \sum_{j=1, j \neq i}^{N} k\left(x_{i}, x_{j}\right) .
$$

If the kernel is symmetric, i.e., $k(x, y)=k(y, x)$ for all $x, y \in X$, we may also write

$$
E\left(\omega_{N}\right)=2 \sum_{1 \leq i<j \leq N} k\left(x_{i}, x_{j}\right) .
$$

For a set $A \subset X$, the $N$-point energy of $A$ is given by

$$
\mathcal{E}(A, N):=\inf \left\{E\left(\omega_{N}\right): \omega_{N} \subset A, \operatorname{card}\left(\omega_{N}\right)=N\right\} .
$$

We say that $\omega_{N}^{*} \subset A$ is an optimal $N$-point configuration on $A$ if

$$
E\left(\omega_{N}^{*}\right)=\mathcal{E}(A, N)
$$

When $A$ is compact, such a configuration always exists by the lower semicontinuity of $k$. In order to study the asymptotic behavior of the sequence $E\left(\omega_{N}^{*}\right)$ we need to introduce the continuous counterparts of the above notions.

Let $\mathcal{M}(A)$ denote the linear space of all real-valued Radon measures that are compactly supported on $A$, and let $\mathcal{M}^{+}(A):=\{\mu \in \mathcal{M}(A): \mu \geq 0\}$. We also introduce the class $\mathcal{M}_{1}(A):=\left\{\mu \in \mathcal{M}^{+}(A): \mu(X)=1\right\}$. Given a measure $\mu \in \mathcal{M}(A)$, the continuous energy of $\mu$ is the double integral

$$
W(\mu):=\iint k(x, y) d \mu(x) d \mu(y) .
$$

The function

$$
U^{\mu}(x):=\int k(x, y) d \mu(y)
$$

is called the potential of $\mu$. Since any l.s.c. function is bounded below on compact sets, the above integrals are well defined, although they may attain the value $+\infty$.

We say that $k$ satisfies the maximum principle if for every measure $\mu \in \mathcal{M}_{1}(A)$,

$$
\sup _{x \in \operatorname{supp}(\mu)} U^{\mu}(x)=\sup _{x \in X} U^{\mu}(x) .
$$

The quantity $w(A):=\inf \left\{W(\mu): \mu \in \mathcal{M}_{1}(A)\right\}$ plays an important role in potential theory and is called the Wiener energy of $A$. The capacity of $A$ is defined as $\operatorname{cap}_{k}(A):=w(A)^{-1}$ if $k$ is positive, and otherwise, it is defined $\operatorname{ascap}_{k}(A):=$ 
$\exp (-w(A))$. A property is said to hold quasi-everywhere (q.e.), if the exceptional set has Wiener energy $+\infty$.

Given a net $\left\{\mu_{\alpha}\right\} \subset \mathcal{M}(A)$, we say that $\left\{\mu_{\alpha}\right\}$ converges in the weak-star topology to a measure $\mu \in \mathcal{M}(A)$ when

$$
\lim _{\alpha} \int f d \mu_{\alpha}=\int f d \mu, \quad \text { for all } f \in C_{c}(A),
$$

where $C_{c}(A)$ denotes the space of compactly supported continuous functions on $A$. We will use the notation

$$
\mu_{\alpha} \stackrel{*}{\longrightarrow} \mu
$$

to denote the weak-star convergence of measures. If $A$ is compact, we know by the Banach-Alaoglu theorem that $\mathcal{M}_{1}(A)$ equipped with the weak-star topology is compact.

If $A$ is compact and $w(A)<\infty$, a measure $\mu \in \mathcal{M}_{1}(A)$ satisfying the property $W(\mu)=w(A)$ is called an equilibrium measure. The existence of such a measure is guaranteed by the lower semicontinuity of $k$ and the compactness of $\mathcal{M}_{1}(A)$ (see Theorem 2.3 in [17]). However, uniqueness does not always hold.

The following result is due to G. Choquet [10, and it is central in this theory.

Theorem 1.1. Let $k$ be an arbitrary kernel and $A \subset X$ a compact set. If $\left\{\omega_{N}^{*}\right\}$ is a sequence of optimal $N$-point configurations on $A$, then

$$
\lim _{N \rightarrow \infty} \frac{E\left(\omega_{N}^{*}\right)}{N^{2}}=w(A)
$$

The following variation of Theorem 1.1 was obtained by Farkas and Nagy [16].

Theorem 1.2. Assume that the kernel $k$ is positive and is finite on the diagonal, i.e., $k(x, x)<+\infty$ for all $x \in X$. Then for arbitrary sets $A \subset X$,

$$
\lim _{N \rightarrow \infty} \frac{\mathcal{E}(A, N)}{N^{2}}=w(A),
$$

where $\mathcal{E}(A, N)$ is the $N$-point energy of $A$ (see (1)).

In this paper we study an alternative construction of points obtained by means of a "greedy" algorithm.

Definition 1.3. Let $k: X \times X \rightarrow \mathbb{R} \cup\{+\infty\}$ be a symmetric kernel on a locally compact metric space $X$, and let $A \subset X$ be a compact set. A sequence $\left(a_{n}\right)_{n=1}^{\infty} \subset A$ is called a greedy $k$-energy sequence on $A$ if it is generated in the following way:

- $a_{1}$ is selected arbitrarily on $A$.

- Assuming that $a_{1}, \ldots, a_{n}$ have been selected, $a_{n+1}$ is chosen to satisfy

$$
\sum_{i=1}^{n} k\left(a_{n+1}, a_{i}\right)=\inf _{x \in A} \sum_{i=1}^{n} k\left(x, a_{i}\right)
$$

for every $n \geq 1$.

We remark that the choice of $a_{n+1}$ is not unique in general. We will use the notation

$$
\alpha_{N, k}:=\left\{a_{1}, \ldots, a_{N}\right\}
$$

to denote the set of the first $N$ points of this sequence. It is significantly easier to obtain numerically these configurations rather than optimal $N$-point configurations, since in order to obtain the former we have to minimize a functional of one variable instead of $N$ variables. 
It was shown by Fuglede (see Theorem 2.4 in [17]) that if $k$ is symmetric and $A \subset$ $X$ is compact, every $\mu \in \mathcal{M}_{1}(A)$ that has minimal energy satisfies the inequality $U^{\mu}(x) \leq w(A)$ for all $x \in \operatorname{supp}(\mu)$. The essential support of $\mu$ is the set

$$
S_{\mu}^{*}:=\left\{x \in A: U^{\mu}(x) \leq w(A)\right\} .
$$

Hence, $\operatorname{supp}(\mu) \subset S_{\mu}^{*}$.

The following is a restricted version of Definition 1.3

Definition 1.4. Under the same assumptions as in Definition 1.3, assume that $w(A)<\infty$, and let $\mu \in \mathcal{M}_{1}(A)$ be an equilibrium measure. A sequence $\left(a_{n}=\right.$ $\left.a_{n, k, \mu}\right)_{n=1}^{\infty} \subset A$ is called a greedy $(k, \mu)$-energy sequence on $A$ if it is generated in the following way:

- $a_{1}$ is selected arbitrarily on $S_{\mu}^{*}$.

- Assuming that $a_{1}, \ldots, a_{n}$ have been selected, $a_{n+1}$ is chosen to satisfy $a_{n+1} \in S_{\mu}^{*}$ and

$$
\sum_{i=1}^{n} k\left(a_{n+1}, a_{i}\right)=\inf _{x \in S_{\mu}^{*}} \sum_{i=1}^{n} k\left(x, a_{i}\right)
$$

for every $n \geq 1$.

The set of the first $N$ points of this sequence is denoted by $\alpha_{N, k, \mu}$.

Albert Edrei [14] was probably the first person who studied the point configurations $\alpha_{N, k}$ in the particular case $X=\mathbb{C}$ and $k(x, y)=-\log |x-y|$. However, in the literature these configurations are often called Leja points, in recognition of Leja's article [23. When the kernel employed is the Green function or the Newtonian kernel $k(x, y)=1 /|x-y|$ in the unit sphere $S^{2}$, the configurations $\alpha_{N, k}$ are also referred to as Leja-Górski points (see [19] and references therein). In [1, certain configurations known as fast Leja points are introduced, and an algorithm is presented to compute them. These configurations are defined over discretizations of planar sets and the kernel employed is the logarithmic kernel. In [11] a constrained energy problem for this kernel is considered and associated constrained Leja points are introduced. We remark that Leja points are important in interpolation theory because they provide a Newton-type interpolation point scheme on the real line or complex plane.

A very relevant class of kernels is the so-called M. Riesz kernels in $X=\mathbb{R}^{p}$, which depend on a parameter $s$ in $[0,+\infty)$. It is defined as follows:

$$
k_{s}(x, y):=K(|x-y| ; s), \quad x, y \in \mathbb{R}^{p},
$$

where $|\cdot|$ denotes the Euclidean norm and

$$
K(t ; s):=\left\{\begin{array}{ccc}
t^{-s}, & \text { if } \quad s>0, \\
-\log (t), & \text { if } \quad s=0 .
\end{array}\right.
$$

We shall use the notations $I_{s}(\mu)$ and $U_{s}^{\mu}$ to denote the energy (2) and potential (3) of a measure $\mu \in \mathcal{M}(A)$ with respect to the Riesz $s$-kernel, and $w_{s}(A)$ to denote the Wiener energy of a set $A$ in this new setting. We will also use $E_{s}\left(\omega_{N}\right)$ to represent the discrete energy of an $N$-point configuration $\omega_{N} \subset \mathbb{R}^{p}$, and

$$
\mathcal{E}_{s}(A, N):=\inf \left\{E_{s}\left(\omega_{N}\right): \omega_{N} \subset A, \operatorname{card}\left(\omega_{N}\right)=N\right\}
$$

to denote the $N$-point Riesz $s$-energy of a compact set $A \subset \mathbb{R}^{p}$. Additionally, greedy $k_{s}$-energy configurations will be denoted by $\alpha_{N, s}$. 
A few words about Riesz $s$-kernels are needed at this point. Let $A \subset \mathbb{R}^{p}$ be compact, and $0 \leq s<\operatorname{dim}_{\mathcal{H}}(A)$, where $\operatorname{dim}_{\mathcal{H}}(A)$ denotes the Hausdorff dimension of $A$ (which will be denoted by $d$ throughout the rest of this section). Then there is a unique equilibrium measure $\lambda_{A, s} \in \mathcal{M}_{1}(A)$ with finite energy, i.e., $I_{s}\left(\lambda_{A, s}\right)=$ $w_{s}(A)<+\infty$. On the other hand, if $s \geq d$, then $I_{s}(\mu)=+\infty$ for all $\mu \in \mathcal{M}_{1}(A)$. We refer the reader to Theorems 8.5 and 8.9 in [26] for justifications of these facts.

For $s<d$, Theorem 1.1 asserts that

$$
\lim _{N \rightarrow \infty} \frac{E_{s}\left(\omega_{N, s}^{*}\right)}{N^{2}}=I_{s}\left(\lambda_{A, s}\right),
$$

where $\left\{\omega_{N, s}^{*}\right\}$ denotes any sequence of optimal $N$-point configurations on $A$ with respect to the Riesz $s$-kernel. In addition (see [22]),

$$
\frac{1}{N} \sum_{x \in \omega_{N, s}^{*}} \delta_{x} \stackrel{*}{\rightarrow} \lambda_{A, s}, \quad N \rightarrow \infty
$$

where $\delta_{x}$ is the Dirac unit measure concentrated at $x$. If $s \geq d$, then Theorem 1.1 tells us that

$$
\lim _{N \rightarrow \infty} \frac{E_{s}\left(\omega_{N, s}^{*}\right)}{N^{2}}=+\infty,
$$

so the order of growth of $E_{s}\left(\omega_{N, s}^{*}\right)$ is greater than $N^{2}$.

Throughout the rest of the paper we denote by $\operatorname{Vol}\left(B^{d}\right)$ the volume of the unit ball $B^{d}$ in $\mathbb{R}^{d}$, and $\mathcal{H}_{d}$ represents $d$-dimensional Hausdorff measure in $\mathbb{R}^{p}$ (normalized by the condition $\mathcal{H}_{d}\left([0,1]^{d}\right)=1$, where $[0,1]^{d}$ denotes here the embedding of the $d$-dimensional unit cube in $\mathbb{R}^{p}$ ). Regarding the case $s \geq d$, in [20] and 4 ] geometric measure theoretic tools were employed to obtain the following result.

Theorem 1.5. Let $A$ be a compact subset of a d-dimensional $C^{1}$-manifold in $\mathbb{R}^{p}$. If $\left\{\omega_{N, d}^{*}\right\}$ is any sequence of optimal $N$-point configurations on $A$ for $s=d$, then

$$
\lim _{N \rightarrow \infty} \frac{E_{d}\left(\omega_{N, d}^{*}\right)}{N^{2} \log N}=\frac{\operatorname{Vol}\left(B^{d}\right)}{\mathcal{H}_{d}(A)} .
$$

Furthermore, if $\mathcal{H}_{d}(A)>0$, any sequence $\left\{\widetilde{\omega}_{N}\right\}$ of configurations on $A$ whose energies satisfy (11) is uniformly distributed with respect to $\mathcal{H}_{d}$ in the sense that

$$
\frac{1}{N} \sum_{x \in \widetilde{\omega}_{N}} \delta_{x} \stackrel{*}{\longrightarrow} \frac{\left.\mathcal{H}_{d}\right|_{A}}{\mathcal{H}_{d}(A)}, \quad N \rightarrow \infty .
$$

Assume now that $A \subset \mathbb{R}^{p}$ is a d-rectifiable compact set, i.e., $A$ is the image of a bounded set in $\mathbb{R}^{d}$ under a Lipschitz mapping. If $\left\{\omega_{N, s}^{*}\right\}$ is any sequence of optimal $N$-point configurations on $A$ for $s>d$, it holds that

$$
\lim _{N \rightarrow \infty} \frac{E_{s}\left(\omega_{N, s}^{*}\right)}{N^{1+s / d}}=\frac{C_{s, d}}{\mathcal{H}_{d}(A)^{s / d}},
$$

where $C_{s, d}>0$ is a constant independent of $A$ and $p$. In addition, if $\mathcal{H}_{d}(A)>0$, any sequence of configurations on $A$ whose energies satisfy (13) is uniformly distributed with respect to $\mathcal{H}_{d}$.

We remark that the constant $C_{s, d}$ equals $2 \zeta(s)$ when $d=1$, where $\zeta(s)$ is the classical Riemann zeta function (cf. [25]). 
Definition 1.6. Let $A$ be a compact set of Hausdorff dimension $d$. A sequence of point sets $\omega_{N} \subset A$, is said to be asymptotically s-energy minimizing on $A$, and we shall write $\left\{\omega_{N}\right\}_{N} \in \operatorname{AEM}(A ; s)$, if it satisfies, with $\omega_{N, s}^{*}$ replaced by $\omega_{N}$, the limit relation (10), (11) or (13), according to whether $s<d, s=d$, or $s>d$.

We conclude this section by illustrating in Figures 14 the first 200 points of four approximate greedy $k_{s}$-energy sequences on the unit square $A=[0,1]^{2}$ for four different values of $s$ (for better visualization we have deleted the coordinate axes). The initial point is always selected to be the origin. The points in Figures 24 were obtained by minimizing over a discretization of $[0,1]^{2}$ formed by the set

$$
\{(i / 100, j / 100): 0 \leq i, j \leq 100\},
$$

whereas, in the case of Figure 1 the points were obtained using a discretization of the boundary of $[0,1]^{2}$ consisting of 4000 equally spaced points. We remark that if $s=0$, it follows from the maximum modulus principle that all greedy energy points will lie on the boundary of the square and thus only the boundary was discretized in this case.

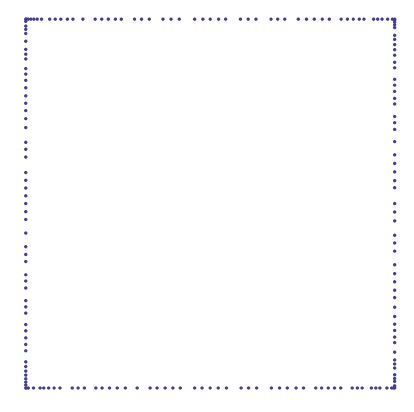

FiguRE 1. $s=0$.

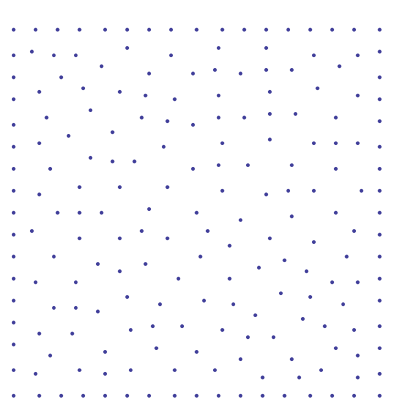

FiguRE 3. $s=2$.

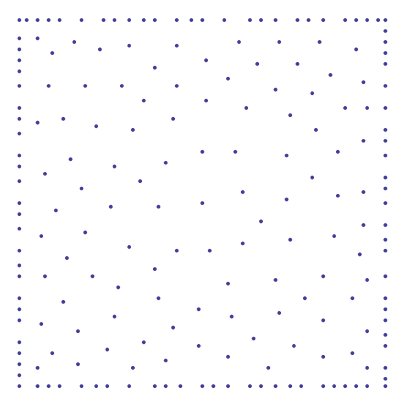

FiguRe 2. $s=1$.

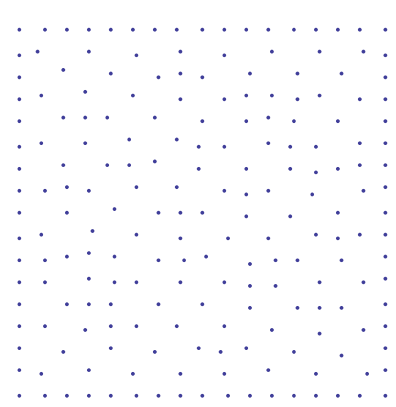

FiguRE $4 . s=4$.

In Section 2 we state and discuss our main results. Their proofs are given in subsequent sections. 


\section{MAin Results}

\subsection{The Potential theoretic case: Sets of positive capacity. Let}

$$
U_{n}(x):=\sum_{j=1}^{n-1} k\left(x, a_{j}\right), \quad n \geq 2 .
$$

Our first result on the asymptotic behavior of greedy sequences is the following.

Theorem 2.1. Let $k: X \times X \rightarrow \mathbb{R} \cup\{+\infty\}$ be a symmetric kernel on a locally compact metric space $X$ that satisfies the maximum principle. Assume $A \subset X$ is a compact set and $\left\{\alpha_{N, k}\right\}$ is a greedy k-energy sequence on $A$. Then

(i) the following limit holds:

$$
\lim _{N \rightarrow \infty} \frac{E\left(\alpha_{N, k}\right)}{N^{2}}=w(A) ;
$$

(ii) if $w(A)<\infty$ and the equilibrium measure $\mu \in \mathcal{M}_{1}(A)$ is unique, it follows that

$$
\frac{1}{N} \sum_{a \in \alpha_{N, k}} \delta_{a} \stackrel{*}{\longrightarrow} \mu, \quad N \rightarrow \infty
$$

(iii) if $w(A)<\infty$, it holds that

$$
\lim _{n \rightarrow \infty} \frac{U_{n}\left(a_{n}\right)}{n}=w(A)
$$

where $a_{n}$ is the $n$-th element of the greedy $k$-energy sequence.

Furthermore, if $w(A)<\infty$, the analogues of assertions ( $i$ ), (ii), and (iii) hold for any greedy $(k, \mu)$-energy sequence on $A$ without assuming the maximum principle.

Theorem 2.1 generalizes a result due to Siciak 30 (see Lemma 3.1) stated for Riesz potentials. For sets of positive capacity, his result asserts that if $A \subset \mathbb{R}^{p}$ is a compact set, $p-2 \leq s<p, p \geq 2$, and $\left\{\alpha_{N, s}\right\}$ is a greedy $k_{s}$-energy sequence on $A$, then (16) holds for $k=k_{s}$.

As a consequence of Theorem 2.1, we deduce the following corollaries for Riesz kernels. Throughout this paper we denote the $d$-dimensional unit sphere in $\mathbb{R}^{d+1}$ by $S^{d}$.

Corollary 2.2. Let $d$ be a positive integer and $s \in[0, d)$. Then any greedy $k_{s}$-energy sequence $\alpha_{N, s} \subset S^{d}$ is $A E M\left(S^{d} ; s\right)$ and the asymptotic formula 1

$$
\lim _{N \rightarrow \infty} \frac{E_{s}\left(\alpha_{N, s}\right)}{N^{2}}=\left\{\begin{array}{ccc}
\frac{\Gamma((d+1) / 2) \Gamma(d-s)}{\Gamma((d-s+1) / 2) \Gamma(d-s / 2)}, & \text { if } & 0<s<d, \\
-\log (2)+\frac{1}{2}(\psi(d)-\psi(d / 2)), & \text { if } & s=0,
\end{array}\right.
$$

holds, where $\psi(x):=\Gamma^{\prime}(x) / \Gamma(x)$ denotes the digamma function. In addition,

$$
\frac{1}{N} \sum_{a \in \alpha_{N, s}} \delta_{a} \stackrel{*}{\longrightarrow} \sigma_{d}, \quad N \rightarrow \infty
$$

where $\sigma_{d}$ is the normalized Lebesgue measure on $S^{d}$.

\footnotetext{
${ }^{1}$ We remark that for $d=1$ and $s=0$ we have $\mathcal{E}_{0}\left(S^{1}, N\right)=-N \log (N), N \geq 2$, (cf. [6]).
} 
Corollary 2.3. Let $\alpha_{N, s}$ be any greedy $k_{s}$-energy sequence on $[-1,1]$. For $s \in[0,1)$, this sequence is $A E M([-1,1] ; s)$, which means that

$$
\lim _{N \rightarrow \infty} \frac{E_{s}\left(\alpha_{N, s}\right)}{N^{2}}=\left\{\begin{array}{clc}
\frac{\sqrt{\pi} \Gamma(1+s / 2)}{\cos (\pi s / 2) \Gamma((1+s) / 2)}, & \text { if } & 0<s<1, \\
\log (2), & \text { if } \quad s=0 .
\end{array}\right.
$$

Furthermore,

$$
\frac{1}{N} \sum_{a \in \alpha_{N, s}} \delta_{a} \stackrel{*}{\longrightarrow} \frac{c_{s}}{\left(1-x^{2}\right)^{(1-s) / 2}} d x, \quad x \in[-1,1], \quad N \rightarrow \infty
$$

where $c_{s}$ is a normalizing constant.

Our next result concerns second-order asymptotics for Riesz energy on the unit circle. It is known that if $s \in(0,1)$, then the following limit holds (see [6]):

$$
\lim _{N \rightarrow \infty} \frac{\mathcal{E}_{s}\left(S^{1}, N\right)-I_{s}(\sigma) N^{2}}{N^{1+s}}=\frac{2 \zeta(s)}{(2 \pi)^{s}}
$$

where $\mathcal{E}_{s}\left(S^{1}, N\right)$ denotes (see (9) ) the $N$-point minimal Riesz $s$-energy of $S^{1}$, and $\zeta(s)$ is the analytic extension of the classical Riemann zeta function. We know by Corollary 2.2 that all greedy $k_{s}$-energy sequences are $\operatorname{AEM}\left(S^{1} ; s\right)$ when $s \in$ $(0,1)$. Nevertheless, the expression (21) below shows that in terms of second-order asymptotics greedy $k_{s}$-energy sequences and optimal $N$-point configurations for $s \in(0,1)$ behave differently.

Proposition 2.4. Let $s \in(0,1)$ and consider an arbitrary greedy $k_{s}$-energy sequence $\left\{\alpha_{N, s}\right\}_{N}$ on $S^{1}$. Then the following next order asymptotics holds:

$$
\lim _{n \rightarrow \infty} \frac{E_{s}\left(\alpha_{3 \cdot 2^{n}, s}\right)-I_{s}(\sigma)\left(3 \cdot 2^{n}\right)^{2}}{\left(3 \cdot 2^{n}\right)^{1+s}}=f(s) \frac{2 \zeta(s)}{(2 \pi)^{s}},
$$

where $f(s)=\frac{1}{2}\left(\frac{4}{3}\right)^{1+s}+\left(\frac{1}{3}\right)^{1+s}<1$ for $s \in(0,1), \zeta(s)$ is the analytic extension of the classical Riemann zeta function, and $\sigma$ is the normalized arclength measure on $S^{1}$.

If $s \in(0,1)$, then $\zeta(s)<0$, and therefore $f(s) \frac{2 \zeta(s)}{(2 \pi)^{s}}>\frac{2 \zeta(s)}{(2 \pi)^{s}}$. Hence, we obtain the following

Corollary 2.5. For all $s \in(0,1)$ and for any greedy $k_{s}$-energy sequence $\left\{\alpha_{N, s}\right\}_{N}$ on $S^{1}$, the sequence

$$
\frac{E_{s}\left(\alpha_{N, s}\right)-I_{s}(\sigma) N^{2}}{N^{1+s}}
$$

is not convergent.

Remark. It is well known that on $S^{1}$ the minimal $N$-point Riesz $s$-energy $\mathcal{E}_{s}\left(S^{1}, N\right)$ is attained only by configurations consisting of $N$ equally spaced points, and this property holds for every $s \geq 0$. It can be shown (see Lemma 4.2) that for such $s$ greedy configurations $\alpha_{2^{n}, s}$ on $S^{1}$ are formed by $2^{n}$ equally spaced points. 


\subsection{Sets of capacity zero.}

2.2.1. Greedy $k_{s}$-energy sequences on $S^{1}$. In this subsection we present some results about the asymptotic behavior of $E_{s}\left(\alpha_{N, s}\right)$ for greedy $k_{s}$-energy sequences on $S^{1}$ when $s \geq 1$. As we shall see in Proposition 2.6, greedy $k_{s}$-energy sequences on $S^{1}$ are not $\operatorname{AEM}\left(S^{1} ; s\right)$ for $s>1$, which is perhaps a surprising result. We conclude that the behavior of $E_{s}\left(\alpha_{N, s}\right)$ exhibits a transition at $s=1$, the Hausdorff dimension of $S^{1}$, since as we saw in the previous section, greedy $k_{s}$-energy sequences are $\operatorname{AEM}\left(S^{1} ; s\right)$ for $s<1$.

Remark. It follows from the geometric lemmas in Section 4 that greedy $k_{s}$-energy sequences $\alpha_{N, s}$ on $S^{1}$ are independent of $s$, i.e., once the points $a_{1}, \ldots, a_{n}$ have been selected, the choice of $a_{n+1}$ is independent of the value of $s$ and depends only on the position of the first $n$ points of the sequence. As a consequence we will denote greedy $k_{s}$-energy sequences on $S^{1}$ by $\alpha_{N}$ instead of $\alpha_{N, s}$.

In 25] (see Theorem 3.1) it was proved that if $\Gamma$ is a rectifiable Jordan arc, then for $s>1$,

$$
\lim _{N \rightarrow \infty} \frac{E_{s}\left(\omega_{N, s}^{*}\right)}{N^{1+s}}=\frac{2 \zeta(s)}{\mathcal{H}_{1}(\Gamma)^{s}}
$$

and if $s=1$,

$$
\lim _{N \rightarrow \infty} \frac{E_{1}\left(\omega_{N, 1}^{*}\right)}{N^{2} \log N}=\frac{2}{\mathcal{H}_{1}(\Gamma)},
$$

where $\left\{\omega_{N, s}^{*}\right\}_{N}$ is any sequence of optimal $N$-point configurations with respect to the Riesz $s$-kernel.

We remind the reader that by $\mathcal{E}_{s}\left(S^{1}, N\right)$ we denote the $N$-point Riesz $s$-energy of $S^{1}$ (see (9)). As it was observed previously, optimal $N$-point configurations on $S^{1}$ consist precisely of $N$ equally spaced points, and this property holds for all values of $s \in[0, \infty)$. From (22) we have

$$
\lim _{N \rightarrow \infty} \frac{\mathcal{E}_{s}\left(S^{1}, N\right)}{N^{1+s}}=\frac{2 \zeta(s)}{(2 \pi)^{s}} .
$$

By Corollary 2.2 and Theorem 2.18 (see Subsection 2.2.3) we know that if $s \in$ $[0, d]$, then any greedy $k_{s}$-energy sequence $\left\{\alpha_{N, s}\right\}$ on $S^{d}$ is $\operatorname{AEM}\left(S^{d} ; s\right)$. However, the situation changes when $s>1$ on $S^{1}$.

Proposition 2.6. For $s>1$, any greedy $k_{s}$-energy sequence $\left\{\alpha_{N, s}\right\}_{N}$ on $S^{1}$ is not asymptotically s-energy minimizing. In fact, the subsequence $\alpha_{3 \cdot 2^{n}, s}$ satisfies

$$
\lim _{n \rightarrow \infty} \frac{E_{s}\left(\alpha_{3 \cdot 2^{n}, s}\right)}{\left(3 \cdot 2^{n}\right)^{1+s}}=f(s) \frac{2 \zeta(s)}{(2 \pi)^{s}}
$$

where $f(s)=\frac{1}{2}\left(\frac{4}{3}\right)^{1+s}+\left(\frac{1}{3}\right)^{1+s}>1$ for all $s>1$.

As in the previous section, we want to describe the difference in terms of secondorder asymptotics between greedy $k_{s}$-energy sequences and optimal $N$-point configurations when $s=1$. The following formula holds (see [6]):

$$
\lim _{N \rightarrow \infty} \frac{\mathcal{E}_{1}\left(S^{1}, N\right)-\frac{1}{\pi} N^{2} \log N}{N^{2}}=\frac{1}{\pi}(\gamma-\log (\pi / 2)),
$$

where $\gamma=\lim _{M \rightarrow \infty}\left(1+\frac{1}{2}+\cdots+\frac{1}{M}-\log M\right)$ denotes the Euler-Mascheroni constant. 
Proposition 2.7. For any greedy $k_{1}$-energy sequence $\left\{\alpha_{N}\right\}_{N}$ on $S^{1}$ we have

$$
\lim _{n \rightarrow \infty} \frac{E_{1}\left(\alpha_{3 \cdot 2^{n}}\right)-\frac{1}{\pi}\left(3 \cdot 2^{n}\right)^{2} \log \left(3 \cdot 2^{n}\right)}{\left(3 \cdot 2^{n}\right)^{2}}=\frac{1}{\pi}\left(\gamma-\log (\pi / 2)+\log \left(2^{\frac{16}{9}} / 3\right)\right) .
$$

Corollary 2.8. For any greedy $k_{1}$-energy sequence $\left\{\alpha_{N}\right\}_{N}$ on $S^{1}$, the sequence

$$
\frac{E_{1}\left(\alpha_{N}\right)-\frac{1}{\pi} N^{2} \log N}{N^{2}}
$$

is not convergent.

2.2.2. $k_{s}$-Energy of sequences on Jordan arcs or curves in $\mathbb{R}^{p}$ for $s \geq 1$ and bestpacking. Throughout this subsection, by a Jordan arc in $\mathbb{R}^{p}$ we understand a set homeomorphic to a closed segment. A closed Jordan curve refers to a set homeomorphic to a circle.

Our main result states that for $s>1$ it is not possible to find any sequence of points on a Jordan arc or curve that is asymptotically $s$-energy minimizing.

Theorem 2.9. Let $\left\{x_{k}\right\}_{k=0}^{\infty} \subset \Gamma$ be an arbitrary sequence of distinct points, where $\Gamma$ is a rectifiable Jordan arc or closed Jordan curve in $\mathbb{R}^{p}$. Set $\mathcal{X}_{n}:=\left\{x_{k}\right\}_{k=0}^{n}$. Then $\left\{\mathcal{X}_{n}\right\}_{n} \notin A E M(\Gamma ; s)$ for all $s>1$. In particular, $\left\{\alpha_{N, s}\right\} \notin A E M(\Gamma ; s)$ for any greedy $k_{s}$-energy sequence on $\Gamma$ when $s>1$.

The next result shows that, in contrast to the case $s>1$, for $s=1$ greedy $k_{1^{-}}$ energy sequences on $S^{1}$ are $\operatorname{AEM}\left(S^{1} ; 1\right)$. More generally, we shall prove this fact for smooth Jordan arcs or curves $\Gamma$ by which we mean that the natural parametrization $\Phi:[0, L] \longrightarrow \Gamma$, where $L=\mathcal{H}_{1}(\Gamma)$, is of class $C^{1}$ and $\Phi^{\prime}(t) \neq \mathbf{0}$ for all $t \in[0, L]$.

Theorem 2.10. Let $\Gamma \subset \mathbb{R}^{p}$ be a smooth Jordan arc or closed curve, and let $s=d=1$. Then any greedy $k_{1}$-energy sequence $\left\{\alpha_{N, 1}\right\}$ on $\Gamma$ is $A E M(\Gamma ; 1)$, i.e.,

$$
\lim _{N \rightarrow \infty} \frac{E_{1}\left(\alpha_{N, 1}\right)}{N^{2} \log N}=\frac{2}{\mathcal{H}_{1}(\Gamma)} .
$$

Furthermore,

$$
\frac{1}{N} \sum_{a \in \alpha_{N, 1}} \delta_{a} \stackrel{*}{\longrightarrow} \frac{\left.\mathcal{H}_{1}\right|_{\Gamma}}{\mathcal{H}_{1}(\Gamma)}, \quad N \rightarrow \infty .
$$

For the analogous result for greedy $k_{d}$-energy on the unit sphere $S^{d} \subset \mathbb{R}^{d+1}$, see Theorem 2.18 .

We next consider best-packing configurations. For a collection of $N$ distinct points $\omega_{N}=\left\{x_{1}, \ldots, x_{N}\right\} \subset \mathbb{R}^{p}$ we set

$$
\delta\left(\omega_{N}\right):=\min _{1 \leq i \neq j \leq N}\left|x_{i}-x_{j}\right|,
$$

and for an infinite set $A \subset \mathbb{R}^{p}$, we let

$$
\delta_{N}(A):=\sup \left\{\delta\left(\omega_{N}\right): \omega_{N} \subset A, \operatorname{card}\left(\omega_{N}\right)=N\right\}
$$

be the best-packing distance of $N$-point configurations on $A$. In $[5]$ it is shown (see Theorem 2.2) that if $A=\Gamma$ is a rectifiable Jordan curve or arc in $\mathbb{R}^{p}$, then

$$
\lim _{N \rightarrow \infty} N \delta_{N}(\Gamma)=\mathcal{H}_{1}(\Gamma) .
$$

This fact leads us to the following. 
Definition 2.11. Let $\Gamma \subset \mathbb{R}^{p}$ be a Jordan arc or curve, and let $\omega_{N} \subset \Gamma$ be a sequence of $N$-point configurations. We say that $\left\{\omega_{N}\right\} \in \operatorname{AEM}(\Gamma, \infty)$ if

$$
\lim _{N \rightarrow \infty} N \delta\left(\omega_{N}\right)=\mathcal{H}_{1}(\Gamma) .
$$

Theorem 2.12. Let $\Gamma \subset \mathbb{R}^{p}$ be a rectifiable Jordan arc or curve with length $L=$ $\mathcal{H}_{1}(\Gamma)$, and let $\left\{x_{k}\right\}_{k=0}^{\infty} \subset \Gamma$ be an arbitrary infinite sequence such that $x_{i} \neq x_{j}$ if $i \neq j$. Set $\mathcal{X}_{n}:=\left\{x_{0}, \ldots, x_{n}\right\}$. Then $\left\{\mathcal{X}_{n}\right\} \notin A E M(\Gamma, \infty)$. In fact,

$$
\liminf _{n \rightarrow \infty} n \delta\left(\mathcal{X}_{n}\right) \leq \frac{4+3 \sqrt{2}}{4+4 \sqrt{2}} L<L .
$$

Moreover, if $c:=\lim \sup _{n \rightarrow \infty} n \delta\left(\mathcal{X}_{n}\right)>\frac{2+\sqrt{2}}{4} L$, then

$$
\liminf _{n \rightarrow \infty} n \delta\left(\mathcal{X}_{n}\right) \leq \frac{L}{2}+\sqrt{c(L-c)}<c .
$$

In particular, if $\limsup _{n \rightarrow \infty} n \delta\left(\mathcal{X}_{n}\right)=L$, then $\liminf _{n \rightarrow \infty} n \delta\left(\mathcal{X}_{n}\right) \leq L / 2$.

In analogy with finite $s$, we define greedy best-packing configurations on a compact set $A \subset \mathbb{R}^{p}$ by selecting $a_{0} \in A$ and choosing $a_{n} \in A$ so that

$$
\min _{0 \leq i \leq n-1}\left|a_{n}-a_{i}\right|=\max _{x \in A} \min _{0 \leq i \leq n-1}\left|x-a_{i}\right| .
$$

Such points are referred to in [12 as Leja-Bos points. Theorem 2.12 shows that such points are not asymptotically optimal on rectifiable Jordan arcs or curves.

In 12 there appears a conjecture attributed to L. Bos stating that if $A$ is a compact domain of $\mathbb{C}$, every Leja-Bos sequence $\left\{a_{n}\right\}_{n=0}^{\infty}$ on $A$ with $\left|a_{0}\right|=\max \{|x|$ : $x \in A\}$ is asymptotically uniformly distributed. We wish to point out that this conjecture is false as the following result asserts (see also Figure 1 in Section 5).

Proposition 2.13. There exist greedy best-packing sequences on $[0,1]$ and $[0,1]^{2}$ that are not asymptotically uniformly distributed.

It is obvious, however, that greedy best-packing sequences are dense in $A$.

2.2.3. Weighted Riesz potentials. In this subsection we will consider the notion of weighted discrete Riesz energy introduced in [4]. We reproduce here the main definitions.

Definition 2.14. Let $A \subset \mathbb{R}^{p}$ be an infinite compact set whose $d$-dimensional Hausdorff measure $\mathcal{H}_{d}(A)$ is finite. A symmetric function $w: A \times A \longrightarrow[0, \infty]$ is called a CPD-weight function on $A \times A$ if

- $w$ is continuous (as a function on $A \times A$ ) at $\mathcal{H}_{d}$-almost every point of the diagonal $D(A):=\{(x, x): x \in A\}$,

- there is some neighborhood $G$ of $D(A)$ (relative to $A \times A$ ) such that $\inf _{G} w>$ 0 , and

- $w$ is bounded on any closed subset $B \subset A \times A$ such that $B \cap D(A)=\emptyset$.

The term CPD stands for (almost) continuous and positive on the diagonal.

Definition 2.15. Let $s>0$. Given a collection of $N(N \geq 2)$ points $\omega_{N}:=$ $\left\{x_{1}, \ldots, x_{N}\right\} \subset A$, the weighted Riesz s-energy of $\omega_{N}$ is defined by

$$
E_{s}^{w}\left(\omega_{N}\right):=\sum_{1 \leq i \neq j \leq N} \frac{w\left(x_{i}, x_{j}\right)}{\left|x_{i}-x_{j}\right|^{s}}
$$


while the $N$-point weighted Riesz s-energy of $A$ is given by

$$
\mathcal{E}_{s}^{w}(A, N):=\inf \left\{E_{s}^{w}\left(\omega_{N}\right): \omega_{N} \subset A, \operatorname{card}\left(\omega_{N}\right)=N\right\} .
$$

The weighted Hausdorff measure $\mathcal{H}_{d}^{s, w}$ on Borel sets $B \subset A$ is defined by

$$
\mathcal{H}_{d}^{s, w}(B):=\int_{B}(w(x, x))^{-d / s} d \mathcal{H}_{d}(x) .
$$

The following result about the asymptotic behavior of $\left\{\mathcal{E}_{s}^{w}(A, N)\right\}_{N}$ was obtained in 4 .

Theorem 2.16. Let $A$ be a compact subset of a d-dimensional $C^{1}$-manifold in $\mathbb{R}^{p}$ and assume that $w: A \times A \rightarrow[0, \infty]$ is a CPD-weight function on $A \times A$. Then

$$
\lim _{N \rightarrow \infty} \frac{\mathcal{E}_{d}^{w}(A, N)}{N^{2} \log N}=\frac{\operatorname{Vol}\left(B^{d}\right)}{\mathcal{H}_{d}^{d, w}(A)} .
$$

Furthermore, if $\mathcal{H}_{d}(A)>0$ and $\left\{\widetilde{\omega}_{N}\right\}$ is a sequence of configurations on $A$ satisfying (31), with $\mathcal{E}_{d}^{w}(A, N)$ replaced by $E_{d}^{w}\left(\widetilde{\omega}_{N}\right)$, then

$$
\frac{1}{N} \sum_{x \in \widetilde{\omega}_{N}}^{N} \delta_{x} \stackrel{*}{\longrightarrow} \frac{\left.\mathcal{H}_{d}^{d, w}\right|_{A}}{\mathcal{H}_{d}^{d, w}(A)}, \quad N \rightarrow \infty .
$$

Assume now that $A \subset \mathbb{R}^{p}$ is a d-rectifiable set. Then for $s>d$,

$$
\lim _{N \rightarrow \infty} \frac{\mathcal{E}_{s}^{w}(A, N)}{N^{1+s / d}}=\frac{C_{s, d}}{\left[\mathcal{H}_{d}^{s, w}(A)\right]^{s / d}}
$$

where $C_{s, d}$ is the same positive constant that appears in Theorem 1.5. In addition, if $\mathcal{H}_{d}(A)>0$, any sequence $\left\{\widetilde{\omega}_{N}\right\}$ of configurations on A satisfying (33) with $\mathcal{E}_{s}^{w}(A, N)$ replaced by $E_{s}^{w}\left(\widetilde{\omega}_{N}\right)$ also satisfies (32).

Definition 2.17. Let $w$ be a lower semicontinuous CPD-weight function on $A \times A$. A sequence $\left(a_{n}\right)_{n=1}^{\infty} \subset A$ is called a greedy $(w, s)$-energy sequence on $A$ if it is generated in the same way as in Definition 1.3, with $k(x, y):=w(x, y) /|x-y|^{s}$.

The next result concerns greedy $(w, d)$-energy points on the unit sphere $S^{d} \subset$ $\mathbb{R}^{d+1}$

Theorem 2.18. Assume that $w: S^{d} \times S^{d} \rightarrow[0, \infty)$ is a continuous function such that $w(x, x)>0$ for all $x \in S^{d}$. Let $\left\{\alpha_{N, d}^{w}\right\}_{N}$ be an arbitrary greedy $(w, d)$-energy sequence on $S^{d}, d \geq 1$. Then

$$
\lim _{N \rightarrow \infty} \frac{E_{d}^{w}\left(\alpha_{N, d}^{w}\right)}{N^{2} \log N}=\frac{\operatorname{Vol}\left(B^{d}\right)}{\mathcal{H}_{d}^{d, w}\left(S^{d}\right)}
$$

and therefore,

$$
\frac{1}{N} \sum_{a \in \alpha_{N, d}^{w}} \delta_{a} \stackrel{*}{\longrightarrow} \frac{\left.\mathcal{H}_{d}^{d, w}\right|_{S^{d}}}{\mathcal{H}_{d}^{d, w}\left(S^{d}\right)}, \quad N \rightarrow \infty .
$$

In particular, any greedy $k_{d}$-energy sequence $\left\{\alpha_{N, d}\right\}_{N}$ on $S^{d}$ is $A E M\left(S^{d}, d\right)$ and satisfies (18) for $s=d$.

In the following result we consider greedy $(w, p)$-energy sequences on sets in $\mathbb{R}^{p}$ with positive Lebesgue measure. 
Theorem 2.19. Let $A \subset \mathbb{R}^{p}$ be a compact set such that $\mathcal{H}_{p}(A)>0$, and let $\left\{\alpha_{N, p}^{w}\right\}_{N}$ be an arbitrary greedy $(w, p)$-energy sequence on $A$. Assume that $w$ : $A \times A \rightarrow[0, \infty)$ is a continuous function such that $w(x, x)>0$ for all $x \in A$. Then

$$
\lim _{N \rightarrow \infty} \frac{E_{p}^{w}\left(\alpha_{N, p}^{w}\right)}{N^{2} \log N}=\frac{\operatorname{Vol}\left(B^{p}\right)}{\mathcal{H}_{p}^{p, w}(A)}
$$

and therefore,

$$
\frac{1}{N} \sum_{a \in \alpha_{N, p}^{w}} \delta_{a} \stackrel{*}{\longrightarrow} \frac{\left.\mathcal{H}_{p}^{p, w}\right|_{A}}{\mathcal{H}_{p}^{p, w}(A)}, \quad N \rightarrow \infty .
$$

In particular, any greedy $k_{p}$-energy sequence $\left\{\alpha_{N, p}\right\}_{N}$ on $A$ is $A E M(A ; p)$ and is asymptotically uniformly distributed with respect to $\mathcal{H}_{p}$.

In view of Proposition 2.6, it is not in general possible to extend Theorem 2.18 to $s>d$. However, for any compact set $A \subset \mathbb{R}^{p}$ with $\mathcal{H}_{\delta}(A)>0$ (where $\delta>0$ is arbitrary, not necessarily an integer), we can show that the order of growth of $E_{s}^{w}\left(\alpha_{N, s}^{w}\right)$ when $s>\delta(s=\delta)$ is at most $N^{1+s / \delta}\left(N^{2} \log N\right)$. Let

$$
\mathcal{H}_{\delta}^{\infty}(A):=\inf \left\{\sum_{i}\left(\operatorname{diam} G_{i}\right)^{\delta}: A \subset \bigcup_{i} G_{i}\right\}, \quad \delta>0 .
$$

Theorem 2.20. Let $0<\delta \leq p$. Assume that $A \subset \mathbb{R}^{p}$ is a compact set such that $\mathcal{H}_{\delta}(A)>0$. Let $w$ be a bounded lower semicontinuous CPD-weight function on $A \times A$. Consider an arbitrary greedy $(w, s)$-energy sequence $\left\{\alpha_{N, s}^{w}\right\}_{N} \subset A$, for $s \geq \delta$. Then, for $N \geq 2$,

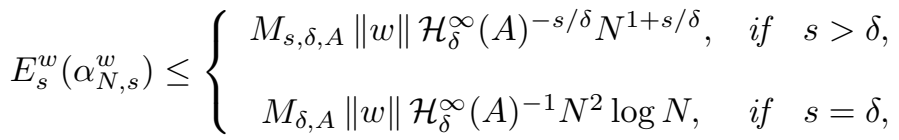

where the constants $M_{s, \delta, A}>0$ and $M_{\delta, A}>0$ are independent of $w$ and $N$, and $\|w\|:=\sup \{w(x, y): x, y \in A\}$.

Corollary 2.21. Let $A \subset \mathbb{R}^{p}$ be a d-rectifiable set. Suppose $s>d$ and $w$ is a bounded lower semicontinuous CPD-weight function on $A \times A$. Consider an arbitrary greedy $(w, s)$-energy sequence $\left\{\alpha_{N, s}^{w}\right\}_{N} \subset A$. Then there are constants $C_{1}, C_{2}>0$ such that

$$
C_{1} N^{1+s / d} \leq E_{s}^{w}\left(\alpha_{N, s}^{w}\right) \leq C_{2} N^{1+s / d} .
$$

If $s=d$ and $A$ is assumed to be a compact subset of a d-dimensional $C^{1}$-manifold, then there are constants $C_{3}, C_{4}>0$ such that

$$
C_{3} N^{2} \log N \leq E_{d}^{w}\left(\alpha_{N, d}^{w}\right) \leq C_{4} N^{2} \log N,
$$

for any greedy $(w, d)$-energy sequence $\left\{\alpha_{N, d}^{w}\right\}_{N} \subset A$.

Corollary 2.22. Let $A \subset \mathbb{R}^{p}$ be a d-rectifiable set. Suppose $s>d$ and $w$ is a bounded lower semicontinuous CPD-weight function on $A \times A$. Consider an arbitrary greedy $(w, s)$-energy sequence $\left\{a_{n}\right\}_{n=1}^{\infty} \subset A$. Then $\left\{a_{n}\right\}_{n=1}^{\infty}$ is dense in $A$. If $s=d$ and $A$ is assumed to be a compact subset of a d-dimensional $C^{1}$-manifold, the same conclusion holds for any greedy $(w, d)$-energy sequence. Taking $w \equiv 1$ the result is applicable to greedy $k_{s}$-energy sequences.

We can slightly improve the density result for the case of an interval. 
Proposition 2.23. Let $[a, b] \subset \mathbb{R}$ and $s>1$. Assume that $w$ is a bounded lower semicontinuous $C P D$-weight function on $[a, b] \times[a, b]$, and $\left(a_{n}\right)_{n=1}^{\infty}$ is a greedy $(w, s)$ energy sequence on $[a, b]$. If $I$ is any closed subinterval of $[a, b]$, then

$$
\liminf _{N \rightarrow \infty} \frac{\left(\operatorname{card}\left\{1 \leq n \leq N: a_{n} \in I\right\}\right)^{1+\frac{1}{s}}}{N}>0 .
$$

We conclude this section by remarking that some results about greedy sequences in the context of external fields have been obtained by the first author and will appear in a separate work.

\section{Proofs of Results from Section 2.1}

Proof of Theorem 2.1. Assume first that $w(A)<\infty, \mu \in \mathcal{M}_{1}(A)$ is an equilibrium measure, and $\left\{\alpha_{N, k, \mu}\right\}$ is an arbitrary greedy $(k, \mu)$-energy sequence on $A$. If $a_{n}$ is the $n$-th element of this sequence, it follows by definition that

$$
U_{n}\left(a_{n}\right) \leq U_{n}(x), \quad \text { for all } \quad x \in S_{\mu}^{*}, \quad n \geq 2 .
$$

Hence, for any $x \in S_{\mu}^{*}$,

$$
\begin{array}{r}
E\left(\alpha_{N, k, \mu}\right)=\sum_{1 \leq i \neq j \leq N} k\left(a_{i}, a_{j}\right)=2 \sum_{j=2}^{N} \sum_{i=1}^{j-1} k\left(a_{i}, a_{j}\right) \\
=2 \sum_{j=2}^{N} U_{j}\left(a_{j}\right) \leq 2 \sum_{j=2}^{N} U_{j}(x)=2 \sum_{j=2}^{N} \sum_{i=1}^{j-1} k\left(x, a_{i}\right) .
\end{array}
$$

We now integrate the above inequality with respect to $\mu$ to obtain

$$
E\left(\alpha_{N, k, \mu}\right) \leq 2 \sum_{j=2}^{N} \sum_{i=1}^{j-1} U^{\mu}\left(a_{i}\right) .
$$

Taking into account that $U^{\mu}\left(a_{i}\right) \leq w(A)$ for all $i\left(a_{i} \in S_{\mu}^{*}\right)$ it follows that

$$
E\left(\alpha_{N, k, \mu}\right) \leq N(N-1) w(A) .
$$

Now, if $\left\{\omega_{N}^{*}\right\}$ is a sequence of optimal $N$-point configurations on $A$, then $E\left(\omega_{N}^{*}\right) \leq$ $E\left(\alpha_{N, k, \mu}\right)$ for all $N$. Therefore (14) for $\alpha_{N, k, \mu}$ is a consequence of (40) and (5).

Consider the sequence of normalized counting measures

$$
\nu_{N}:=\frac{1}{N} \sum_{a \in \alpha_{N, k, \mu}} \delta_{a}
$$

and assume that the equilibrium measure $\mu$ is unique. Let $g_{n}: A \times A \rightarrow \mathbb{R}$ be a non-decreasing sequence of continuous functions that converges pointwise to $k$ on $A \times A$ (recall that $k$ is lower semicontinuous and hence lower bounded on $A \times A$ ). 
We have

$$
\begin{gathered}
\iint g_{n}(x, y) d \nu_{N}(x) d \nu_{N}(y)=\frac{1}{N^{2}} \sum_{i=1}^{N} \sum_{j=1}^{N} g_{n}\left(a_{i}, a_{j}\right) \\
=\frac{1}{N^{2}}\left(\sum_{i=1}^{N} g_{n}\left(a_{i}, a_{i}\right)+\sum_{1 \leq i \neq j \leq N} g_{n}\left(a_{i}, a_{j}\right)\right) \\
\leq \frac{1}{N^{2}}\left(\sum_{i=1}^{N} g_{n}\left(a_{i}, a_{i}\right)+\sum_{1 \leq i \neq j \leq N} k\left(a_{i}, a_{j}\right)\right) ;
\end{gathered}
$$

hence

$$
\iint g_{n}(x, y) d \nu_{N}(x) d \nu_{N}(y) \leq \frac{\sum_{i=1}^{N} g_{n}\left(a_{i}, a_{i}\right)+E\left(\alpha_{N, k, \mu}\right)}{N^{2}} .
$$

By the compactness of $A$ and the continuity of $g_{n}$, there exists a constant $M_{n}>0$ such that

$$
\sum_{i=1}^{N}\left|g_{n}\left(a_{i}, a_{i}\right)\right| \leq N M_{n} .
$$

Therefore, for each fixed $n$ we have

$$
\lim _{N \rightarrow \infty} \frac{\sum_{i=1}^{N} g_{n}\left(a_{i}, a_{i}\right)+E\left(\alpha_{N, k, \mu}\right)}{N^{2}}=w(A) .
$$

Now let $\left\{\nu_{N}\right\}_{N \in \mathcal{N}}$ be a subsequence that converges in the weak-star topology to a measure $\lambda \in \mathcal{M}_{1}(A)$. Since $\nu_{N} \times \nu_{N}$ converges weak-star to $\lambda \times \lambda$, we have

$$
\lim _{N \rightarrow \infty} \iint g_{n}(x, y) d \nu_{N}(x) d \nu_{N}(y)=\iint g_{n}(x, y) d \lambda(x) d \lambda(y) .
$$

Thus from (41) and (42) we conclude that

$$
\iint g_{n}(x, y) d \lambda(x) d \lambda(y) \leq w(A)
$$

and letting $n \rightarrow \infty$ we obtain

$$
W(\lambda)=\iint k(x, y) d \lambda(x) d \lambda(y) \leq w(A) .
$$

It follows that $\lambda$ is an equilibrium measure. By hypothesis there is only one equilibrium measure. Thus $\lambda=\mu$ and (15) is proved for $\alpha_{N, k, \mu}$.

We next show (16) for $\alpha_{N, k, \mu}$. It is not assumed now that the equilibrium measure is unique, and $\alpha_{N, k, \mu}$ denotes a greedy $(k, \mu)$-energy sequence associated with a certain equilibrium measure $\mu$. We know from the first part of the proof that

$$
\lim _{N \rightarrow \infty} \frac{E\left(\alpha_{N, k, \mu}\right)}{N^{2}}=\lim _{N \rightarrow \infty} \frac{2 \sum_{i=2}^{N} U_{i}\left(a_{i}\right)}{N^{2}}=w(A) .
$$

For every $n \geq 1$,

$$
\frac{U_{n+1}\left(a_{n+1}\right)}{n}=\inf _{x \in S_{\mu}^{*}} \frac{1}{n} \sum_{i=1}^{n} k\left(x, a_{i}\right) .
$$


Integrating this equality with respect to $\mu$ and recalling that $a_{i} \in S_{\mu}^{*}$, we get

$$
\frac{U_{n+1}\left(a_{n+1}\right)}{n} \leq \frac{1}{n} \sum_{i=1}^{n} \int k\left(x, a_{i}\right) d \mu(x)=\frac{1}{n} \sum_{i=1}^{n} U^{\mu}\left(a_{i}\right) \leq w(A) .
$$

On the other hand, for every $n \geq 2$,

$$
U_{n+1}\left(a_{n+1}\right) \geq U_{n}\left(a_{n}\right)+L
$$

where $L:=\inf \left\{k(x, y): x, y \in S_{\mu}^{*}\right\}$. By rescaling $k$ we may assume that $L \leq-1$.

Let $\epsilon \in(0,1)$. Assume that $m$ is an integer such that

$$
\frac{U_{m+1}\left(a_{m+1}\right)}{m}<w(A)-\epsilon .
$$

We shall show that this inequality can hold only for finitely many integers $m$. Applying (45) repeatedly we obtain for $(1+\epsilon /(3 L)) m \leq i \leq m$,

$$
\frac{U_{i+1}\left(a_{i+1}\right)}{m} \leq w(A)-\epsilon-\frac{(m-i) L}{m} \leq w(A)-\epsilon+\frac{\epsilon / 3}{1+\epsilon /(3 L)} \leq w(A)-\frac{\epsilon}{2},
$$

and so

$$
\frac{U_{i+1}\left(a_{i+1}\right)}{i} \leq \frac{m}{i}(w(A)-\epsilon / 2) \leq \frac{m}{i} w(A)-\frac{\epsilon}{2} .
$$

Taking into account (44) and the last inequality, we get

$$
\begin{aligned}
& \frac{2}{(m+1) m} \sum_{i=1}^{m} U_{i+1}\left(a_{i+1}\right) \leq \frac{2}{(m+1) m} \sum_{1 \leq i<(1+\epsilon /(3 L)) m} i w(A) \\
& +\frac{2}{(m+1) m} \sum_{(1+\epsilon /(3 L)) m \leq i \leq m} m w(A)-\frac{\epsilon}{2} \frac{2}{(m+1) m} \sum_{(1+\epsilon /(3 L)) m \leq i \leq m} i .
\end{aligned}
$$

Furthermore, it is easy to see that

$$
\begin{gathered}
-\frac{\epsilon}{2} \frac{2}{(m+1) m} \sum_{(1+\epsilon /(3 L)) m \leq i \leq m} i \leq \frac{\epsilon^{2}}{6 L(m+1)}\left(1+2 m+\frac{m \epsilon}{3 L}\right) \\
\leq \frac{\epsilon^{2}(1+\epsilon /(3 L))}{6 L} .
\end{gathered}
$$

If $w(A) \leq 0$, then

$$
\frac{2}{(m+1) m}\left\{\sum_{1 \leq i<(1+\epsilon /(3 L)) m} i w(A)+\sum_{(1+\epsilon /(3 L)) m \leq i \leq m} m w(A)\right\} \leq w(A)
$$

and hence it follows from (47) and (48) that

$$
\frac{2}{(m+1) m} \sum_{i=1}^{m} U_{i+1}\left(a_{i+1}\right) \leq w(A)+\frac{\epsilon^{2}(1+\epsilon /(3 L))}{6 L} .
$$

Since the right-hand side of (49) is a constant strictly less than $w(A)$, by (43) it follows that there are only finitely many integers $m$ satisfying (46). This implies with (44) that (16) holds. 
Now assume that $w(A)>0$. It is easy to verify that

$$
\begin{gathered}
\frac{2}{(m+1) m}\left\{\sum_{1 \leq i<(1+\epsilon /(3 L)) m} i w(A)+\sum_{(1+\epsilon /(3 L)) m \leq i \leq m} m w(A)\right\} \\
\leq\left(1+\frac{2}{m+1}+\frac{\epsilon}{3 L(m+1)}+\frac{\epsilon^{2} m}{9(m+1) L^{2}}\right) w(A),
\end{gathered}
$$

and so, from (47) and (48), we deduce that

$$
\begin{aligned}
& \frac{2}{(m+1) m} \sum_{i=1}^{m} U_{i+1}\left(a_{i+1}\right) \leq\left(1+\frac{2}{m+1}+\frac{\epsilon}{3 L(m+1)}+\frac{\epsilon^{2} m}{9(m+1) L^{2}}\right) w(A) \\
& +\frac{\epsilon^{2}(1+\epsilon /(3 L))}{6 L} .
\end{aligned}
$$

If we assume that there are infinitely many integers $m$ satisfying (46), then applying the last inequality we obtain

$$
\limsup _{N \rightarrow \infty} \frac{2 \sum_{i=2}^{N} U_{i}\left(a_{i}\right)}{N^{2}} \leq w(A)+\frac{\epsilon^{2} w(A)}{9 L^{2}}+\frac{\epsilon^{2}(1+\epsilon /(3 L))}{6 L} .
$$

Without loss of generality assume that $L \leq-1$ also satisfies $L<-(1+2 w(A)) / 3$. Then the right-hand side of (50) is a constant strictly less than $w(A)$, which contradicts (43). This concludes the proof of (16) for $\alpha_{N, k, \mu}$.

If $k$ satisfies the maximum principle, we know by Fuglede's result (see paragraph after Definition (1.3) and (4) that $U^{\mu}(x) \leq w(A)$ for all $x \in A$. Therefore, the assertions (14)-(16) follow (replacing $S_{\mu}^{*}$ by $A$ ) for any greedy $k$-energy sequence $\left\{\alpha_{N, k}\right\}$ on $A$ by using the argument presented above.

Proof of Corollary 2.2. It is well known (see for example [22]) that for any $s<d$ the equilibrium measure associated with the Riesz kernel $k_{s}$ is unique and coincides with $\sigma_{d}$. Since $\operatorname{supp}\left(\sigma_{d}\right)=S^{d}$, any greedy $k_{s}$-energy sequence $\left\{\alpha_{N, s}\right\}_{N} \subset S^{d}$ is a greedy $\left(k_{s}, \sigma_{d}\right)$-energy sequence. Therefore by (14) we obtain that $\left\{\alpha_{N, s}\right\}_{N} \in$ $\operatorname{AEM}\left(S^{d} ; s\right)$. The values on the right-hand side of (17) are the values of $I_{s}\left(\sigma_{d}\right)$. The case $s>0$ follows from formula (1.2) of [21] and the case $s=0$ from formula (2.26) of [8. Finally (18) follows from (15).

Proof of Corollary 2.3. It is shown in 22] that for $s<1$ the equilibrium measure associated with the Riesz kernel $k_{s}$ is

$$
\frac{c_{s}}{\left(1-x^{2}\right)^{(1-s) / 2}} d x, \quad x \in(-1,1),
$$

and its energy is given by the value on the right-hand side of (19).

Proof of Proposition 2.4. We have

$$
\begin{aligned}
& \frac{E_{s}\left(\alpha_{3 \cdot 2^{n}, s}\right)-I_{s}(\sigma)\left(3 \cdot 2^{n}\right)^{2}}{\left(3 \cdot 2^{n}\right)^{1+s}} \\
& =\frac{1}{3^{1+s}} \frac{E_{s}\left(\alpha_{3 \cdot 2^{n}, s}\right)-I_{s}(\sigma)\left(2^{n}\right)^{2}-I_{s}(\sigma) 2^{2 n+3}}{\left(2^{n}\right)^{1+s}} .
\end{aligned}
$$

As will be justified in Section 4 (see Lemma 4.3), the relation

$$
E_{s}\left(\alpha_{3 \cdot 2^{n}, s}\right)=\frac{1}{2} \mathcal{E}_{s}\left(S^{1}, 2^{n+2}\right)+\mathcal{E}_{s}\left(S^{1}, 2^{n}\right)
$$


holds. Therefore, from (51), it follows that

$$
\begin{aligned}
& \frac{E_{s}\left(\alpha_{3 \cdot 2^{n}, s}\right)-I_{s}(\sigma)\left(3 \cdot 2^{n}\right)^{2}}{\left(3 \cdot 2^{n}\right)^{1+s}} \\
& \quad=\frac{1}{3^{1+s}}\left(\frac{\mathcal{E}_{s}\left(S^{1}, 2^{n}\right)-I_{s}(\sigma)\left(2^{n}\right)^{2}}{\left(2^{n}\right)^{1+s}}+\frac{4^{1+s}}{2} \frac{\mathcal{E}_{s}\left(S^{1}, 2^{n+2}\right)-I_{s}(\sigma)\left(2^{n+2}\right)^{2}}{\left(2^{n+2}\right)^{1+s}}\right) .
\end{aligned}
$$

Now applying (20) we get

$$
\lim _{n \rightarrow \infty} \frac{E_{s}\left(\alpha_{3 \cdot 2^{n}, s}\right)-I_{s}(\sigma)\left(3 \cdot 2^{n}\right)^{2}}{\left(3 \cdot 2^{n}\right)^{1+s}}=\left(\frac{1}{2}\left(\frac{4}{3}\right)^{1+s}+\left(\frac{1}{3}\right)^{1+s}\right) \frac{2 \zeta(s)}{(2 \pi)^{s}} .
$$

Finally, it is easy to check that $f(s)=\frac{1}{2}\left(\frac{4}{3}\right)^{1+s}+\left(\frac{1}{3}\right)^{1+s}<1$ for all $s \in(0,1)$.

Proof of Corollary 2.5. Since $\alpha_{2^{n}, s}$ consists of $2^{n}$ equally spaced points (see Lemma 4.2 below), $E_{s}\left(\alpha_{2^{n}, s}\right)=\mathcal{E}_{s}\left(S^{1}, 2^{n}\right)$, and therefore,

$$
\lim _{n \rightarrow \infty} \frac{E_{s}\left(\alpha_{2^{n}, s}\right)-I_{s}(\sigma) 2^{2 n}}{2^{n(1+s)}}=\frac{2 \zeta(s)}{(2 \pi)^{s}},
$$

but the subsequence $\left\{\alpha_{3 \cdot 2^{n}, s}\right\}_{n}$ provides a different limit value, given by (21).

\section{Proofs of Results from Subsection 2.2.1}

In order to prove Proposition 2.6 we need some auxiliary lemmas that give a geometric description of greedy $k_{s}$-energy sequences on $S^{1}$. The proofs of Lemmas 4.1 and 4.2 below are straightforward; cf. 24] for the details.

Lemma 4.1. Let $s \geq 0$ and consider two points $x_{1}, x_{2} \in S^{1}$. Set

$$
f(x):=K\left(\left|x-x_{1}\right| ; s\right)+K\left(\left|x-x_{2}\right| ; s\right), \quad x \in S^{1},
$$

where $K$ is defined in (8). Then on each arc determined by $x_{1}$ and $x_{2}$ the function $f$ has only one minimum and it is attained at the midpoint of the arc.

Lemma 4.2. Let $s \geq 0$ and assume that $\left(a_{n}\right)_{n=1}^{\infty}$ is an arbitrary greedy $k_{s}$-energy sequence on $S^{1}$. Then

(i) for every positive integer $m$, the set $\alpha_{2^{m}, s}$ consists of $2^{m}$ equally spaced points, that is,

$$
\alpha_{2^{m}, s}=\left\{a_{1} e^{i \frac{2 \pi n}{2^{m}}}\right\}_{n=1}^{2^{m}} ;
$$

(ii) for every positive integer $m$, the set $\alpha_{3 \cdot 2^{m}}$ can be written as

$$
\alpha_{3 \cdot 2^{m}}=S_{2^{m+2}} \backslash S_{2^{m}},
$$

where $S_{2^{m+2}}$ and $S_{2^{m}}$ are formed, respectively, by $2^{m+2}$ and $2^{m}$ equally spaced points, and $S_{2^{m}} \subset S_{2^{m+2}}$;

(iii) the choice of any point $a_{n}$ is independent of $s$.

Since greedy $k_{s}$-energy sequences $\left\{\alpha_{N, s}\right\}$ on the unit circle $S^{1}$ are independent of $s$, we will denote them simply by $\alpha_{N}$.

Lemma 4.3. Let $s \geq 0$. Then given any greedy $k_{s}$-energy sequence $\left\{\alpha_{N}\right\}_{N}$ on $S^{1}$ the following relation holds for every $n \geq 1$ :

$$
E_{s}\left(\alpha_{3 \cdot 2^{n}}\right)=\frac{1}{2} \mathcal{E}_{s}\left(S^{1}, 2^{n+2}\right)+\mathcal{E}_{s}\left(S^{1}, 2^{n}\right) .
$$


Proof. If $\left\{x_{k}\right\}_{k=1}^{N} \subset S^{1}$ is an arbitrary collection of $N$ equally spaced points, then using the simple equality $\left|e^{i \xi}-e^{i \theta}\right|=2\left|\sin \left(\frac{\xi-\theta}{2}\right)\right|$, we conclude that for $s>0$,

$$
\mathcal{E}_{s}\left(S^{1}, N\right)=E_{s}\left(\left\{x_{k}\right\}_{k=1}^{N}\right)=2^{-s} N \sum_{n=1}^{N-1} \sin \left(\frac{\pi n}{N}\right)^{-s} .
$$

Consider any greedy $k_{s}$-energy sequence $\left(\alpha_{N}\right)_{N=1}^{\infty}$ on $S^{1}$. We claim that

$$
E_{s}\left(\alpha_{3 \cdot 2^{n}}\right)=E_{s}\left(S_{2^{n+2}}\right)-2^{n+1} \cdot 2^{-s} \sum_{k=1}^{2^{n+2}-1} \sin \left(\frac{\pi k}{2^{n+2}}\right)^{-s}+E_{s}\left(S_{2^{n}}\right),
$$

where $\alpha_{3 \cdot 2^{n}}=S_{2^{n+2}} \backslash S_{2^{n}}$ is as in (52). To see this, notice that $E_{s}\left(\alpha_{3 \cdot 2^{n}}\right)$ is obtained by removing twice from $E_{s}\left(S_{2^{n+2}}\right)$ all terms $\left|e^{i \xi}-e^{i \theta}\right|^{-s}$ where either $e^{i \xi} \in S_{2^{n}}$ or $e^{i \theta} \in S_{2^{n}}$.

Since

$$
E_{s}\left(S_{2^{n+2}}\right)=\mathcal{E}_{s}\left(S^{1}, 2^{n+2}\right), \quad E_{s}\left(S_{2^{n}}\right)=\mathcal{E}_{s}\left(S^{1}, 2^{n}\right),
$$

(53) follows by applying (54). The case $s=0$ is proved similarly.

Proof of Proposition 2.6. Using (53) we obtain

$$
\frac{E_{s}\left(\alpha_{3 \cdot 2^{n}}\right)}{3^{1+s} 2^{n(1+s)}}=\frac{1}{3^{1+s}} \frac{1}{2} \frac{2^{(n+2)(1+s)}}{2^{n(1+s)}} \frac{\mathcal{E}_{s}\left(S^{1}, 2^{n+2}\right)}{2^{(n+2)(1+s)}}+\frac{1}{3^{1+s}} \frac{\mathcal{E}_{s}\left(S^{1}, 2^{n}\right)}{2^{n(1+s)}} .
$$

Simplifying the above expression and applying (24) we conclude that

$$
\lim _{n \rightarrow \infty} \frac{E_{s}\left(\alpha_{3 \cdot 2^{n}}\right)}{\left(3 \cdot 2^{n}\right)^{1+s}}=\left(\frac{1}{2}\left(\frac{4}{3}\right)^{1+s}+\left(\frac{1}{3}\right)^{1+s}\right) \frac{2 \zeta(s)}{(2 \pi)^{s}} .
$$

It is straightforward to check that $f(s)=\frac{1}{2}\left(\frac{4}{3}\right)^{1+s}+\left(\frac{1}{3}\right)^{1+s}>1$ for all $s>1$.

Proof of Proposition 2.7. First observe that

$$
\begin{aligned}
& \frac{E_{1}\left(\alpha_{3 \cdot 2^{n}}\right)-\frac{1}{\pi}\left(3 \cdot 2^{n}\right)^{2} \log \left(3 \cdot 2^{n}\right)}{\left(3 \cdot 2^{n}\right)^{2}} \\
& \quad=\frac{1}{9}\left(\frac{(1 / 2) \mathcal{E}_{1}\left(S^{1}, 2^{n+2}\right)+\mathcal{E}_{1}\left(S^{1}, 2^{n}\right)-\frac{1}{\pi}\left(3 \cdot 2^{n}\right)^{2} \log \left(3 \cdot 2^{n}\right)}{2^{2 n}}\right) .
\end{aligned}
$$

We add and subtract $(1 / \pi) 2^{2 n} \log \left(2^{n}\right)$ to obtain

$$
\begin{gathered}
\frac{E_{1}\left(\alpha_{3 \cdot 2^{n}}\right)-\frac{1}{\pi}\left(3 \cdot 2^{n}\right)^{2} \log \left(3 \cdot 2^{n}\right)}{\left(3 \cdot 2^{n}\right)^{2}} \\
=\frac{1}{9}\left(\frac{\mathcal{E}_{1}\left(S^{1}, 2^{n}\right)-\frac{1}{\pi} 2^{2 n} \log \left(2^{n}\right)}{2^{2 n}}+16 \frac{(1 / 2) \mathcal{E}_{1}\left(S^{1}, 2^{n+2}\right)-\frac{1}{\pi} \Lambda_{n}}{2^{2(n+2)}}\right)
\end{gathered}
$$

where $\Lambda_{n}=\left(3 \cdot 2^{n}\right)^{2} \log \left(3 \cdot 2^{n}\right)-2^{2 n} \log \left(2^{n}\right)$. Taking into account that

$$
\Lambda_{n}=\frac{2^{2(n+2)}}{2} \log \left(2^{n+2}\right)+\log (3)\left(3 \cdot 2^{n}\right)^{2}-8 \log (4) 2^{2 n},
$$

it follows that

$$
\begin{aligned}
16 & \frac{(1 / 2) \mathcal{E}_{1}\left(S^{1}, 2^{n+2}\right)-\frac{1}{\pi} \Lambda_{n}}{2^{2(n+2)}} \\
& =8 \frac{\mathcal{E}_{1}\left(S^{1}, 2^{n+2}\right)-\frac{1}{\pi} 2^{2(n+2)} \log \left(2^{n+2}\right)}{2^{2(n+2)}}+\frac{1}{\pi}(8 \log (4)-9 \log (3)) .
\end{aligned}
$$


Applying (25), (55) and (56) we conclude that

$$
\begin{aligned}
& \lim _{n \rightarrow \infty} \frac{E_{1}\left(\alpha_{3 \cdot 2^{n}}\right)-\frac{1}{\pi}\left(3 \cdot 2^{n}\right)^{2} \log \left(3 \cdot 2^{n}\right)}{\left(3 \cdot 2^{n}\right)^{2}} \\
& \quad=\frac{1}{\pi}(\gamma-\log (\pi / 2))+\frac{1}{\pi}\left(\frac{8}{9} \log (4)-\log (3)\right)=\frac{1}{\pi}\left(\gamma-\log (\pi / 2)+\log \left(2^{\frac{16}{9}} / 3\right)\right) .
\end{aligned}
$$

Proof of Corollary 2.8, Since $E_{1}\left(\alpha_{2^{n}}\right)=\mathcal{E}_{1}\left(S^{1}, 2^{n}\right)$ for all $n$, the result follows from (25) and (26).

\section{Proofs of Results from SUbSection 2.2.2}

Proof of Theorem 2.9. Assume first that $\Gamma$ is a Jordan arc. If $x_{1}, x_{2} \in \Gamma$, we denote by $\left(x_{1}, x_{2}\right)$ the subarc joining $x_{1}$ and $x_{2}$, and by $l\left(x_{1}, x_{2}\right)$ its length.

Let $\mathcal{X}_{n}:=\left\{x_{k, n}\right\}_{k=0}^{n}$ be a sequence of configurations on $\Gamma$, where we assume that the points $x_{k, n}$ are located in successive order. Set

$$
d_{k, n}:=l\left(x_{k-1, n}, x_{k, n}\right), \quad k=1, \ldots, n .
$$

In 25] the following result was proved:

Theorem 5.1. Let $\Gamma$ be a rectifiable Jordan arc in $\mathbb{R}^{p}$. If $s>1$ and $\left\{\mathcal{X}_{n}\right\}_{n} \in$ $A E M(\Gamma ; s)$, then

$$
\lim _{n \rightarrow \infty} \sum_{k=1}^{n}\left|d_{k, n}-\frac{L}{n}\right|=0, \quad L:=\mathcal{H}_{1}(\Gamma)
$$

We prove Theorem 2.9 by contradiction. Let $\left\{x_{k}\right\}_{k=0}^{\infty} \subset \Gamma$ be an arbitrary sequence of distinct points and set $\mathcal{X}_{n}:=\left\{x_{k}\right\}_{k=0}^{n}$. We will use the notation $\mathcal{X}_{n}=\left\{x_{0, n}, \ldots, x_{n, n}\right\}$. Assume that $\left\{\mathcal{X}_{n}\right\}_{n} \in \operatorname{AEM}(\Gamma ; s)$. Let $\delta>0$ and consider the sets

$$
A_{n}^{\delta}:=\left\{k: \in\{1, \ldots, n\}: \frac{L-\delta}{n}<d_{k, n}<\frac{L+\delta}{n}\right\}, \quad B_{n}^{\delta}:=\{1, \ldots, n\} \backslash A_{n}^{\delta} .
$$

Let $\epsilon>0$ be a fixed number. Then from (58) there exists $N=N(\epsilon) \in \mathbb{N}$ such that, if $n \geq N$,

$$
\sum_{k=1}^{n}\left|d_{k, n}-\frac{L}{n}\right| \leq \epsilon
$$

If $k \in B_{n}^{\delta}$, then $\left|d_{k, n}-L / n\right| \geq \delta / n$, and from (59) it follows that

$$
\operatorname{card}\left(B_{n}^{\delta}\right) \frac{\delta}{n} \leq \epsilon, \quad n \geq N
$$

Therefore,

$$
\operatorname{card}\left(A_{n}^{\delta}\right)=n-\operatorname{card}\left(B_{n}^{\delta}\right) \geq n\left(1-\frac{\epsilon}{\delta}\right), \quad n \geq N .
$$

There are exactly $n$ subarcs $\left(x_{k-1, n}, x_{k, n}\right)$, and when we add the next $n / 2$ points (we may assume that $n$ is even) to the configuration $\mathcal{X}_{n}$, obviously at most $n / 2$ of these new points will lie in the subarcs $\left(x_{k-1, n}, x_{k, n}\right)$ where $k \in A_{n}^{\delta}$. Setting

$$
C_{n}^{\delta}:=\left\{k \in A_{n}^{\delta}:\left(x_{k-1, n}, x_{k, n}\right) \text { does not contain a new point }\right\},
$$

we have

$$
\operatorname{card}\left(C_{n}^{\delta}\right) \geq n\left(1-\frac{\epsilon}{\delta}\right)-\frac{n}{2}=n\left(\frac{1}{2}-\frac{\epsilon}{\delta}\right) .
$$


Now since the intervals $\left(x_{k-1, n}, x_{k, n}\right)$ with $k \in C_{n}^{\delta}$ do not contain a new point, there are at least $\operatorname{card}\left(C_{n}^{\delta}\right)$ values of $k^{\prime}$ in $\{1, \ldots, 3 n / 2\}$ such that $d_{k^{\prime}, 3 n / 2}=d_{k, n}$ for some $k \in C_{n}^{\delta}$. For these values of $k^{\prime}$ and the corresponding values of $k$, we have

$$
\left|d_{k^{\prime}, 3 n / 2}-\frac{L}{3 n / 2}\right|=\left|d_{k, n}-\frac{L}{n}+\frac{L}{3 n}\right| .
$$

Now we choose $\delta$ to be any fixed value less than $L / 3$, say $\delta:=L / 6$. Then for $k \in C_{n}^{\delta}$,

$$
\left|d_{k, n}-\frac{L}{n}+\frac{L}{3 n}\right| \geq\left|\frac{L}{3 n}-\right| \frac{L}{n}-d_{k, n}||=\frac{L}{3 n}-\left|\frac{L}{n}-d_{k, n}\right|>\frac{L}{3 n}-\frac{L}{6 n}=\frac{L}{6 n} .
$$

Finally,

$$
\sum_{k^{\prime}=1}^{3 n / 2}\left|d_{k^{\prime}, 3 n / 2}-\frac{L}{3 n / 2}\right| \geq n\left(\frac{1}{2}-\frac{\epsilon}{\delta}\right) \frac{L}{6 n}=\left(\frac{1}{2}-\frac{6 \epsilon}{L}\right) \frac{L}{6} .
$$

But the above estimate contradicts (59) since we can select $\epsilon$ sufficiently small so that

$$
\left(\frac{1}{2}-\frac{6 \epsilon}{L}\right) \frac{L}{6}>\epsilon
$$

If $\Gamma$ is a closed Jordan curve, we select an orientation for it. Then the above reasoning used to prove the result in the case of Jordan arcs is also applicable. We only have to define $\left(x_{k-1, n}, x_{k, n}\right)$ as the subarc joining $x_{k-1, n}$ and $x_{k, n}$ on which a particle moves from $x_{k-1, n}$ to $x_{k, n}$ following the orientation prescribed. The details of the argument are left to the reader.

Proof of Theorem 2.10. We first assume that $\Gamma$ is a smooth Jordan arc of length $L$. We will reduce the problem of asymptotics of $\alpha_{N, 1}$ on $\Gamma$ to a weighted problem on $[0, L]$ and then apply Theorem 2.19 , Let $\Phi:[0, L] \longrightarrow \Gamma$ be the natural parametrization of $\Gamma$ and define $w:[0, L] \times[0, L] \longrightarrow[0, \infty)$ by

$$
w(x, y):=\frac{|x-y|}{|\Phi(x)-\Phi(y)|} .
$$

Let $\Psi=\Phi^{-1}$ be the inverse function of $\Phi$. If $a_{n}$ is the $n$-th element of the greedy $k_{1}$-energy sequence on $\Gamma$, let $b_{n}:=\Psi\left(a_{n}\right) \in[0, L]$ and $\beta_{N}:=\left\{b_{1}, \ldots, b_{N}\right\}$. Since for $t=\Phi(x), x \in[0, L]$,

$$
\inf _{t \in \Gamma} \sum_{i=1}^{n-1} \frac{1}{\left|t-a_{i}\right|}=\inf _{x \in[0, L]} \sum_{i=1}^{n-1} \frac{1}{\left|\Phi(x)-\Phi\left(b_{i}\right)\right|}=\inf _{x \in[0, L]} \sum_{i=1}^{n-1} \frac{w\left(x, b_{i}\right)}{\left|x-b_{i}\right|},
$$

it follows that $\left\{\beta_{N}\right\}$ is a greedy $(w, 1)$-energy sequence on $[0, L]$ (see Definition 2.17) associated with the weight function (60). Notice that

$$
\mathcal{H}_{1}^{1, w}([0, L])=\int_{0}^{L} w(x, x)^{-1} d x=\int_{0}^{L}\left|\Phi^{\prime}(x)\right| d x=L .
$$

Applying Theorem 2.19 we obtain that

$$
\lim _{N \rightarrow \infty} \frac{E_{1}\left(\alpha_{N, 1}\right)}{N^{2} \log N}=\lim _{N \rightarrow \infty} \frac{E_{1}^{w}\left(\beta_{N}\right)}{N^{2} \log N}=\frac{2}{\mathcal{H}_{1}^{1, w}([0, L])}=\frac{2}{L} .
$$

If $\Gamma$ is a smooth Jordan closed curve and $\Phi:[0, L] \longrightarrow \Gamma$ is the natural parametrization of $\Gamma\left(\Phi(0)=\Phi(L), \Phi^{\prime}(0)=\Phi^{\prime}(L)\right)$, we set

$$
\bar{w}(z, \xi):=\frac{|z-\xi|}{|\Phi(x)-\Phi(y)|}, \quad z=e^{2 \pi i x / L}, \xi=e^{2 \pi i y / L} ; \quad x, y \in[0, L],
$$


and apply (with the aid of Theorem 2.18) a similar argument as above on the unit circle $S^{1}$.

In both cases, (28) is a consequence of (27) and Theorem 1.5.

Proof of Theorem 2.12. Let $p>1$ be a rational number and let $n \in \mathbb{Z}_{+}$be such that $n / p$ is an integer. We denote the first $n+1$ points of the sequence $\left\{x_{k}\right\}_{k=0}^{\infty}$ by $\mathcal{X}_{n}=\left\{x_{0, n}, \ldots, x_{n, n}\right\}$, where as in the proof of Theorem 2.9] the points $x_{k, n}$ are located on $\Gamma$ in successive order. There are exactly $n$ subarcs $\left(x_{i, n}, x_{i+1, n}\right)$. We add to $\mathcal{X}_{n}$ the next $n / p$ points of the sequence $\left\{x_{k}\right\}$. Then there are at least $(p-1) n / p$ subarcs $\left(x_{i, n}, x_{i+1, n}\right)$ not containing a new point. These subarcs have length at least $\delta\left(\mathcal{X}_{n}\right)$. We select $(p-1) n / p$ of those.

On the other hand, there are $2 n / p$ subarcs $\left(x_{i,(p+1) n / p}, x_{i+1,(p+1) n / p}\right)$ remaining with length at least $\delta\left(\mathcal{X}_{(p+1) n / p}\right)$. Consequently,

$$
\frac{(p-1) n}{p} \delta\left(\mathcal{X}_{n}\right)+\frac{2 n}{p} \delta\left(\mathcal{X}_{(p+1) n / p}\right) \leq L .
$$

Thus

$$
\liminf _{n \rightarrow \infty} n \delta\left(\mathcal{X}_{n}\right) \leq \frac{p^{2}+p}{p^{2}+2 p-1} L .
$$

Letting $f(p)$ denote the right-hand side of (62), we see that for $p>1$ the function $f$ attains its minimum when $p=1+\sqrt{2}$, and $f(1+\sqrt{2})=\frac{4+3 \sqrt{2}}{4+4 \sqrt{2}} L$, which establishes (29).

Let $\mathcal{X}_{n_{k}}$ be a subsequence of configurations such that $\lim _{k \rightarrow \infty} n_{k} \delta\left(\mathcal{X}_{n_{k}}\right)=c$. Notice that we cannot apply (61) directly because we cannot assume that $n_{k} / p$ is an integer. Let $\lfloor x\rfloor$ denote the integral part of $x$ and let $\{x\}:=x-\lfloor x\rfloor$. Then we get

$$
\left(n_{k}-\left\lfloor\frac{n_{k}}{p}\right\rfloor\right) \delta\left(\mathcal{X}_{n_{k}}\right)+2\left\lfloor\frac{n_{k}}{p}\right\rfloor \delta\left(\mathcal{X}_{n_{k}+\left\lfloor n_{k} / p\right\rfloor}\right) \leq L .
$$

Since

$$
\left|\left(n_{k}-\left\lfloor\frac{n_{k}}{p}\right\rfloor\right) \delta\left(\mathcal{X}_{n_{k}}\right)-\frac{(p-1)}{p} n_{k} \delta\left(\mathcal{X}_{n_{k}}\right)\right|=\left\{\frac{n_{k}}{p}\right\} \delta\left(\mathcal{X}_{n_{k}}\right) \leq \delta\left(\mathcal{X}_{n_{k}}\right),
$$

it follows that

$$
\lim _{k \rightarrow \infty}\left(n_{k}-\left\lfloor\frac{n_{k}}{p}\right\rfloor\right) \delta\left(\mathcal{X}_{n_{k}}\right)=\frac{(p-1)}{p} c .
$$

Similarly,

$$
\left|(p+1)\left\lfloor\frac{n_{k}}{p}\right\rfloor \delta\left(\mathcal{X}_{n_{k}+\left\lfloor n_{k} / p\right\rfloor}\right)-\left(n_{k}+\left\lfloor\frac{n_{k}}{p}\right\rfloor\right) \delta\left(\mathcal{X}_{n_{k}+\left\lfloor n_{k} / p\right\rfloor}\right)\right| \leq p \delta\left(\mathcal{X}_{n_{k}+\left\lfloor n_{k} / p\right\rfloor}\right)
$$

and thus

$$
\liminf _{k \rightarrow \infty}\left(n_{k}+\left\lfloor\frac{n_{k}}{p}\right\rfloor\right) \delta\left(\mathcal{X}_{n_{k}+\left\lfloor n_{k} / p\right\rfloor}\right)=\liminf _{k \rightarrow \infty}(p+1)\left\lfloor\frac{n_{k}}{p}\right\rfloor \delta\left(\mathcal{X}_{n_{k}+\left\lfloor n_{k} / p\right\rfloor}\right) .
$$

Since $\liminf \operatorname{in}_{n \rightarrow \infty} n \delta\left(\mathcal{X}_{n}\right) \leq \liminf _{k \rightarrow \infty}\left(n_{k}+\left\lfloor n_{k} / p\right\rfloor\right) \delta\left(\mathcal{X}_{n_{k}+\left\lfloor n_{k} / p\right\rfloor}\right)$, we obtain from (63) - 65) that

$$
\frac{2}{p+1} \liminf _{n \rightarrow \infty} n \delta\left(\mathcal{X}_{n}\right) \leq L-\frac{p-1}{p} c .
$$

Therefore,

$$
\liminf _{n \rightarrow \infty} n \delta\left(\mathcal{X}_{n}\right) \leq g(p):=\left(1+\frac{1}{p}\right) \frac{p(L-c)+c}{2} .
$$


If $c=L$ we get immediately that $\liminf _{n \rightarrow \infty} n \delta\left(\mathcal{X}_{n}\right) \leq L / 2$. The function $g$ attains a minimum for $p=\sqrt{c /(L-c)}$ and takes the value $L / 2+\sqrt{c(L-c)}$ at this point. This proves (30).

Proof of Proposition 2.13, Consider the sequence $\left\{a_{n}\right\}_{n=0}^{\infty} \subset[0,1]$ defined as follows:

- $a_{0}:=1, a_{1}:=0, a_{2}:=1 / 2$.

- Assuming that the first $2^{n}+1$ points have been selected, let $a_{2^{n}+i}:=$ $(2 i-1) / 2^{n+1}, 1 \leq i \leq 2^{n}$.

Obviously, $\left\{a_{n}\right\}_{n=0}^{\infty}$ is a greedy best-packing sequence on $[0,1]$. However, the sequence of configurations $S_{N}:=\left\{a_{n}\right\}_{n=0}^{N}$ is not uniformly distributed since

$$
\lim _{n \rightarrow \infty} \frac{\operatorname{card}\left(S_{3 \cdot 2^{n-1}} \cap[0,1 / 2]\right)}{3 \cdot 2^{n-1}+1}=\lim _{n \rightarrow \infty} \frac{2^{n}+1}{3 \cdot 2^{n-1}+1}=\frac{2}{3} \neq \frac{1}{2} .
$$

Now we consider the sequence $\left\{b_{n}\right\}_{n=1}^{\infty} \subset[0,1]^{2}$ formed in the following way:

1) $b_{1}:=(1,1), b_{2}:=(0,0), b_{3}:=(0,1), b_{4}:=(1,0)$.

2) Assume that the first $\left(2^{n-1}+1\right)^{2}, n \geq 1$, points have been selected.

2.1) We define the next $2^{2(n-1)}$ points as the centers of the $2^{2(n-1)}$ squares of area $2^{-2(n-1)}$ whose vertices are the points $b_{1}, \ldots, b_{\left(2^{n-1}+1\right)^{2}}$. These $2^{2(n-1)}$ points are chosen in an arbitrary order.

2.2) Now we select the next $2^{n}\left(2^{n-1}+1\right)$ points to be the middle points of the edges of the $2^{2(n-1)}$ squares mentioned above. The first group of points that we add consists of those points with abscissa equal to 0 . The second group is formed by those with abscissa equal to $2^{-n}$. In general, the points from the $i$-th group have abscissa $(i-1) / 2^{n}$. We add exactly $2^{n}+1$ groups, and in each one of them, the points are selected in an arbitrary order.

Figure [5 illustrates the first 221 points of the sequence $\left\{b_{n}\right\}_{n=1}^{\infty}$.

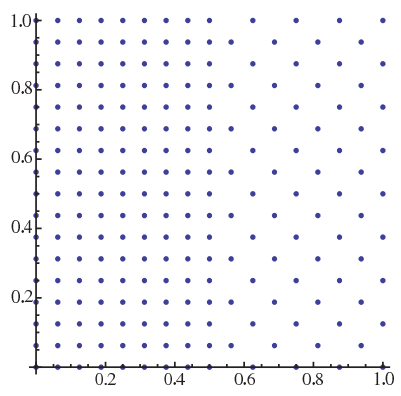

FiguRE 5. Greedy best-packing points for a square: a counterexample to a conjecture of Bos.

Using Voronoi cell decompositions one can show that $\left\{b_{n}\right\}_{n=1}^{\infty}$ is a greedy bestpacking sequence on $[0,1]^{2}$. Indeed, if we consider this Voronoi decomposition of $[0,1]^{2}$ corresponding to the points $\left\{b_{i}\right\}_{1}^{N}$, that is, $[0,1]^{2}=\bigcup_{i=1}^{N} V_{i}$ where

$$
V_{i}=\left\{x \in[0,1]^{2}:\left|x-b_{i}\right| \leq\left|x-b_{j}\right| \text { for all } j=1, \ldots, N\right\},
$$


then it is easy to see that each $V_{i}$ is a convex polygon with 3,4 or 5 sides and that $b_{N+1}$ corresponds to a vertex of the $V_{i}$ 's that is of maximal distance from the points $\left\{b_{i}\right\}_{i=1}^{N}$.

To show that the sequence of configurations $T_{N}:=\left\{b_{i}\right\}_{i=1}^{N}$ is not asymptotically uniformly distributed, we consider the subsequence of sets consisting of $N(n)=$ $3 \cdot 2^{2(n-1)}+7 \cdot 2^{n-2}+1$ points. We have that

$$
\lim _{n \rightarrow \infty} \frac{\operatorname{card}\left(T_{N(n)} \cap[0,1 / 2] \times[0,1]\right)}{N(n)}=\lim _{n \rightarrow \infty} \frac{\left(2^{n-1}+1\right)\left(2^{n}+1\right)}{N(n)}=\frac{2}{3} \neq \frac{1}{2} .
$$

Using a similar argument it is possible to construct a greedy best-packing sequence on $[0,1]^{p} \subset \mathbb{R}^{p}$ that is not asymptotically uniformly distributed.

We remark that it is still plausible that for any infinite compact $A \subset \mathbb{R}^{p}$ there exists at least one greedy best-packing sequence that is asymptotically uniformly distributed on $A$.

\section{Proofs of Results from SUbSection 2.2.3}

Proof of Theorem 2.18. Given a point $x \in S^{d}$, we define $C(x, r):=\left\{y \in S^{d}\right.$ : $|y-x| \leq r\}$. If $\sigma_{d}$ denotes the normalized Lebesgue measure on $S^{d}$, then the following estimates hold (see formulas (3.7) and (3.4) in [21]):

$$
\begin{gathered}
\int_{S^{d} \backslash C(x, r)} \frac{1}{|x-y|^{d}} d \sigma_{d}(y)=\gamma_{d} \log \left(\frac{1}{r}\right)+\mathcal{O}(1), \quad r \rightarrow 0, \\
\sigma_{d}(C(x, r)) \leq \frac{1}{d} \gamma_{d} r^{d}, \quad d \geq 2,
\end{gathered}
$$

where

$$
\gamma_{d}:=\frac{\Gamma((d+1) / 2)}{\Gamma(1 / 2) \Gamma(d / 2)}
$$

If $d=1$, inequality (67) is not valid since $\sigma_{1}(C(x, r))=\frac{2}{\pi} \arcsin \left(\frac{r}{2}\right)$, but instead we have

$$
\sigma_{1}(C(x, r))=\gamma_{1} r+\mathcal{O}\left(r^{3}\right), \quad r \rightarrow 0 .
$$

For $x \in S^{d}$ and $r>0$,

$$
\mathcal{H}_{d}^{d, w}(C(x, r))=\int_{C(x, r)} w(y, y)^{-1} d \mathcal{H}_{d}(y)=\mathcal{H}_{d}\left(S^{d}\right) \int_{C(x, r)} w(y, y)^{-1} d \sigma_{d}(y) .
$$

Thus

$$
\begin{gathered}
\mathcal{H}_{d}^{d, w}(C(x, r)) \leq \frac{M \mathcal{H}_{d}\left(S^{d}\right) \gamma_{d} r^{d}}{d}, \quad d \geq 2, \\
\mathcal{H}_{1}^{1, w}(C(x, r)) \leq M \mathcal{H}_{1}\left(S^{1}\right) \gamma_{1} r+\mathcal{O}\left(r^{3}\right), \quad r \rightarrow 0,
\end{gathered}
$$

where $M:=\sup \left\{w(y, y)^{-1}: y \in S^{d}\right\}$.

Let $r \in(0,1)$ be fixed and set

$$
D_{i}(r):=S^{d} \backslash C\left(a_{i}, r N^{-\frac{1}{d}}\right), \quad D^{N}(r):=\bigcap_{i=1}^{N} D_{i}(r),
$$


where $a_{i}$ is the $i$-th element of the greedy $(w, d)$-energy sequence. From (70) and (171) we obtain that

$$
\begin{gathered}
\mathcal{H}_{d}^{d, w}\left(D^{N}(r)\right) \geq \mathcal{H}_{d}^{d, w}\left(S^{d}\right)-\frac{M \mathcal{H}_{d}\left(S^{d}\right) \gamma_{d} r^{d}}{d}, \quad d \geq 2, \\
\mathcal{H}_{1}^{1, w}\left(D^{N}(r)\right) \geq \mathcal{H}_{1}^{1, w}\left(S^{1}\right)-M \mathcal{H}_{1}\left(S^{1}\right) \gamma_{1} r+\mathcal{O}\left(\frac{r^{3}}{N^{2}}\right), \quad N \rightarrow \infty .
\end{gathered}
$$

We may assume that the expressions in the right-hand side of the above inequalities are positive since we can take $r$ sufficiently close to 0 and $N$ sufficiently large (we will eventually let $r \rightarrow 0$ and $N \rightarrow \infty$ ).

Let $\epsilon>0$. Since the function $w(x, y) / w(x, x)$ is uniformly continuous on $S^{d} \times S^{d}$, there exists $\delta>0$ such that

$$
\left|\frac{w(x, y)}{w(x, x)}-1\right|<\epsilon, \quad \text { for } \quad|x-y|<\delta .
$$

Consider the function

$$
U_{n, d}^{w}(x):=\sum_{i=1}^{n-1} \frac{w\left(x, a_{i}\right)}{\left|x-a_{i}\right|^{d}}, \quad x \in S^{d}, \quad n \geq 2 .
$$

From the definition of a greedy $(w, d)$-energy sequence we know that $U_{n, d}^{w}\left(a_{n}\right) \leq$ $U_{n, d}^{w}(x)$ for all $x \in S^{d}$. Let $2 \leq n \leq N$ and assume that $r<\delta$. Then $C\left(a_{i}, r N^{-\frac{1}{d}}\right) \subset$ $C\left(a_{i}, \delta\right)$ for all $1 \leq i \leq n-1$ and so

$$
\begin{aligned}
& \int_{D^{N}(r)} U_{n, d}^{w}(x) d \mathcal{H}_{d}^{d, w}(x) \leq \sum_{i=1}^{n-1} \int_{D_{i}(r)} \frac{w\left(x, a_{i}\right)}{w(x, x)} \frac{d \mathcal{H}_{d}(x)}{\left|x-a_{i}\right|^{d}} \\
& \quad \leq \sum_{i=1}^{n-1}\left(\int_{C\left(a_{i}, \delta\right) \backslash C\left(a_{i}, r N^{-\frac{1}{d}}\right)} \frac{1+\epsilon}{\left|x-a_{i}\right|^{d}} d \mathcal{H}_{d}(x)+\int_{S^{d} \backslash C\left(a_{i}, \delta\right)} \frac{w\left(x, a_{i}\right)}{w(x, x)} \frac{d \mathcal{H}_{d}(x)}{\left|x-a_{i}\right|^{d}}\right) \\
& \quad \leq(n-1)\left((1+\epsilon) \mathcal{H}_{d}\left(S^{d}\right) \int_{S^{d} \backslash C\left(a_{i}, r N^{\left.-\frac{1}{d}\right)}\right.} \frac{1}{\left|x-a_{i}\right|^{d}} d \sigma_{d}(x)+C(w, \delta)\right),
\end{aligned}
$$

where $C(w, \delta)$ is some constant depending on $\delta$ and $w$. Using (66) it follows that

$$
\int_{D^{N}(r)} U_{n, d}^{w}(x) d \mathcal{H}_{d}^{d, w}(x) \leq(n-1)(1+\epsilon) \mathcal{H}_{d}\left(S^{d}\right)\left(\frac{\gamma_{d}}{d} \log N-\gamma_{d} \log r+\mathcal{O}(1)\right) .
$$

Therefore,

$$
\begin{gathered}
E_{d}^{w}\left(\alpha_{N, d}^{w}\right)=2 \sum_{n=2}^{N} U_{n, d}^{w}\left(a_{n}\right) \leq 2 \sum_{n=2}^{N} \frac{1}{\mathcal{H}_{d}^{d, w}\left(D^{N}(r)\right)} \int_{D^{N}(r)} U_{n, d}^{w}(x) d \mathcal{H}_{d}^{d, w}(x) \\
\leq \frac{N(N-1)}{\mathcal{H}_{d}^{d, w}\left(D^{N}(r)\right)}(1+\epsilon) \mathcal{H}_{d}\left(S^{d}\right)\left(\frac{\gamma_{d}}{d} \log N-\gamma_{d} \log r+\mathcal{O}(1)\right) .
\end{gathered}
$$

Consequently, from (172) and (73) we get that for $d \geq 1$,

$$
\limsup _{N \rightarrow \infty} \frac{E_{d}^{w}\left(\alpha_{N, d}^{w}\right)}{N^{2} \log N} \leq \frac{1}{\mathcal{H}_{d}^{d, w}\left(S^{d}\right)-\frac{M \mathcal{H}_{d}\left(S^{d}\right) \gamma_{d} r^{d}}{d}}(1+\epsilon) \mathcal{H}_{d}\left(S^{d}\right) \frac{\gamma_{d}}{d} .
$$

After letting $r \rightarrow 0$ and $\epsilon \rightarrow 0$ we obtain that

$$
\limsup _{N \rightarrow \infty} \frac{E_{d}^{w}\left(\alpha_{N, d}^{w}\right)}{N^{2} \log N} \leq \frac{\mathcal{H}_{d}\left(S^{d}\right) \gamma_{d}}{\mathcal{H}_{d}^{d, w}\left(S^{d}\right) d}=\frac{\operatorname{Vol}\left(B^{d}\right)}{\mathcal{H}_{d}^{d, w}\left(S^{d}\right)} .
$$


Finally, since $\mathcal{E}_{d}^{w}\left(S^{d}, N\right) \leq E_{d}^{w}\left(\alpha_{N, d}^{w}\right)$ for all $N$, applying (31) it follows that

$$
\lim _{N \rightarrow \infty} \frac{E_{d}^{w}\left(\alpha_{N, d}^{w}\right)}{N^{2} \log N}=\frac{\operatorname{Vol}\left(B^{d}\right)}{\mathcal{H}_{d}^{d, w}\left(S^{d}\right)} .
$$

The statement about the weak-star convergence of the normalized counting measure associated with $\alpha_{N, d}^{w}$ is also an application of Theorem 2.16.

Remark. It is not difficult to see that greedy $k_{s}$-energy sequences on $S^{d} \subset \mathbb{R}^{d+1}$ satisfy the following property for any $s \in[0, \infty)$. If $\left\{a_{n}\right\}_{n=1}^{\infty}$ denotes such a sequence, then for each integer $m \geq 1$, the choice of $a_{2 m}$ is unique and $a_{2 m}=-a_{2 m-1}$.

It is also easily seen that on $S^{2}$ the configuration formed by the first six points of any greedy $k_{s}$-energy sequence does not depend on $s$ and is a rotation of the configuration $\{(1,0,0),(-1,0,0),(0,1,0),(0,-1,0),(0,0,1),(0,0,-1)\}$ (cf. [24]).

Proof of Theorem 2.19. If $R:=\operatorname{diam}(A)$ is the diameter of $A, r<R$ and $x \in A$, then

$$
\int_{A \backslash B(x, r)} \frac{1}{|x-y|^{p}} d y \leq \int_{B(x, R) \backslash B(x, r)} \frac{1}{|x-y|^{p}} d y=\mathcal{H}_{p-1}\left(S^{p-1}\right) \log (R / r) .
$$

Defining

$$
D_{i}(r):=A \backslash B\left(a_{i}, r N^{-\frac{1}{p}}\right), \quad D^{N}(r):=\bigcap_{i=1}^{N} D_{i}(r),
$$

where $a_{i}$ is the $i$-th element of the greedy $(w, p)$-energy sequence, the proof of Theorem 2.18 is applicable here and yields the result. For instance, using (76) the expression similar to (75) is

$$
\int_{D^{N}(r)} U_{n, p}^{w}(x) d \mathcal{H}_{p}^{p, w}(x) \leq(n-1)(1+\epsilon) \mathcal{H}_{p-1}\left(S^{p-1}\right)\left(\frac{1}{p} \log N-\log r+\mathcal{O}(1)\right) .
$$

Since $\operatorname{Vol}\left(B^{p}\right)=p^{-1} \mathcal{H}_{p-1}\left(S^{p-1}\right)$, (35) follows from (77) and Theorem 2.16. The limit (36) is a consequence of (35) and Theorem 2.16.

Proof of Theorem 2.20. We follow closely the argument on page 20 of [4. The following result is known as Frostman's lemma (see [26]).

Lemma 6.1. Let $\delta>0$ and $A$ be a Borel set in $\mathbb{R}^{p}$. Then $\mathcal{H}_{\delta}(A)>0$ if and only if there exists $\mu \in \mathcal{M}^{+}(A)$ such that $\mu(A)>0$ and

$$
\mu(B(x, r)) \leq r^{\delta}, \quad x \in \mathbb{R}^{p}, \quad r>0,
$$

where $B(x, r)$ denotes the open ball centered at $x$ and radius $r$. Furthermore, one can select $\mu$ so that $\mu(A) \geq c_{p, \delta} \mathcal{H}_{\delta}^{\infty}(A)$, where $c_{p, \delta}$ is independent of $A$.

Let $\mu$ be a measure from Lemma 6.1 and set $r_{0}:=(\mu(A) / 2 N)^{1 / \delta}$. Define the sets

$$
D_{j}:=B\left(a_{j}, r_{0}\right), \quad \mathcal{D}_{N}:=A \backslash \bigcup_{j=1}^{N-1} D_{j},
$$

where $a_{j}$ denotes the $j$-th element of the greedy $(w, s)$-energy sequence. Then, using (78),

$$
\mu\left(\mathcal{D}_{N}\right) \geq \mu(A)-\sum_{j=1}^{N-1} \mu\left(D_{j}\right) \geq \mu(A)-(N-1) r_{0}^{\delta}>\frac{\mu(A)}{2}>0 .
$$


Consider the function $U_{N, s}^{w}$ defined in (74). From (79) we obtain

$$
\begin{aligned}
& U_{N, s}^{w}\left(a_{N}\right) \leq \frac{1}{\mu\left(\mathcal{D}_{N}\right)} \int_{\mathcal{D}_{N}} U_{N, s}^{w}(x) d \mu(x) \leq \frac{2}{\mu(A)} \sum_{j=1}^{N-1} \int_{\mathcal{D}_{N}} \frac{w\left(x, a_{j}\right)}{\left|x-a_{j}\right|^{s}} d \mu(x) \\
& \leq \frac{2\|w\|}{\mu(A)} \sum_{j=1}^{N-1} \int_{A \backslash D_{j}} \frac{1}{\left|x-a_{j}\right|^{s}} d \mu(x),
\end{aligned}
$$

where $\|w\|:=\sup \{w(x, y): x, y \in A\}$. Set $R:=\operatorname{diam}(A)$. Then $\mu(A) \leq R^{\delta}$ by (78). If $y \in A$ and $r \in(0, R]$, then

$$
\begin{aligned}
& \int_{A \backslash B(y, r)} \frac{1}{|x-y|^{s}} d \mu(x) \leq \int_{0}^{r^{-s}} \mu\left(\left\{x \in A: \frac{1}{|x-y|^{s}}>t\right\}\right) d t \\
& \quad \leq \frac{\mu(A)}{R^{s}}+\int_{R^{-s}}^{r^{-s}} \mu\left(B\left(y, t^{-1 / s}\right)\right) d t \leq R^{\delta-s}+\int_{R^{-s}}^{r^{-s}} t^{-\delta / s} d t \\
& \quad \leq\left\{\begin{array}{cll}
R^{\delta-s}+\frac{s}{s-\delta} r^{\delta-s}, & \text { if } \quad s>\delta, \\
1+\delta \log \left(\frac{R}{r}\right), & \text { if } \quad s=\delta .
\end{array}\right.
\end{aligned}
$$

Therefore, for $s>\delta$ we obtain

$$
U_{N, s}^{w}\left(a_{N}\right) \leq \frac{2\|w\|}{\mu(A)}(N-1)\left(R^{\delta-s}+\frac{s}{s-\delta} r_{0}^{1-s / \delta}\right) \leq C_{1}\|w\|\left(\frac{N}{\mu(A)}\right)^{s / \delta},
$$

where $C_{1}>0$ is a constant independent of $N$ and $w$. If $s=\delta$, then

$$
U_{N, \delta}^{w}\left(a_{N}\right) \leq \frac{2\|w\|}{\mu(A)}(N-1)\left(1+\delta \log \left(\frac{R}{r_{0}}\right)\right) \leq C_{2}\|w\|\left(\frac{N \log N}{\mu(A)}\right),
$$

where $C_{2}>0$ is also independent of $N$ and $w$. The sequence $\left\{U_{i, s}^{w}\left(a_{i}\right)\right\}_{N}$ is nondecreasing since

$$
U_{i+1, s}^{w}\left(a_{i+1}\right) \geq U_{i, s}^{w}\left(a_{i}\right)+\frac{w\left(a_{i+1}, a_{i}\right)}{\left|a_{i+1}-a_{i}\right|^{s}}, \quad i \geq 1 .
$$

Therefore, applying $\mu(A) \geq c_{p, \delta} \mathcal{H}_{\delta}^{\infty}(A)$ and (80) and (81), Theorem 2.20 readily follows from

$$
E_{s}^{w}\left(\alpha_{N, s}^{w}\right)=2 \sum_{i=2}^{N} U_{i, s}^{w}\left(a_{i}\right) .
$$

Proof of Corollary 2.21. Since $E_{s}^{w}\left(\alpha_{N, s}^{w}\right) \geq \mathcal{E}_{s}^{w}(A, N)$ for every $N$ and $s \geq d$, the lower bounds in (37) and (38) follow from (33) and (31), respectively. The upper bounds follow from Theorem 2.20 .

Proof of Corollary 2.22, Assume the existence of a point $a \in A$ and $\epsilon>0$ such that $\left\{a_{n}\right\}_{n=1}^{\infty} \cap B(a, \epsilon)=\emptyset$. Let $\alpha_{N, s}^{w}=\left\{a_{1}, \ldots, a_{N}\right\}$. Then

$$
E_{s}^{w}\left(\alpha_{N, s}^{w}\right)=2 \sum_{1 \leq i<j \leq N} \frac{w\left(a_{i}, a_{j}\right)}{\left|a_{i}-a_{j}\right|^{s}} \leq 2 \sum_{j=2}^{N} \sum_{i=1}^{j-1} \frac{w\left(a_{i}, x\right)}{\left|a_{i}-x\right|^{s}},
$$

where the last inequality is valid for any $x \in A$. In particular, taking $x=a$ we get

$$
E_{s}^{w}\left(\alpha_{N, s}^{w}\right) \leq \frac{\|w\|}{\epsilon^{s}} N(N-1)
$$


where $\|w\|=\sup \{w(x, y): x, y \in A\}$. This inequality contradicts the first inequalities in (37) and (38).

Proof of Proposition 2.23. Assume that there exists a subinterval $I=[c, d] \subset[a, b]$ for which (39) is not satisfied. Let $N_{l}$ be a subsequence such that

$$
\lim _{l \rightarrow \infty} \frac{\left(\operatorname{card}\left\{1 \leq n \leq N_{l}: a_{n} \in I\right\}\right)^{1+\frac{1}{s}}}{N_{l}}=0 .
$$

Select $\epsilon>0$ sufficiently small so that $J=[c+\epsilon / 2, d-\epsilon / 2] \subset I$ is not empty. If we define $\nu_{l}:=\operatorname{card}\left\{1 \leq n \leq N_{l}: a_{n} \in J\right\}$, then there exists a subinterval of $J$ of length at least $(d-c-\epsilon) /\left(\nu_{l}+1\right)$ not containing any point from $\left\{a_{n} \in J: 1 \leq n \leq N_{l}\right\}$. Let $x_{l}$ be the center of such a subinterval. We have, for $\alpha_{N_{l}, s}^{w}=\left\{a_{1}, \ldots, a_{N_{l}}\right\}$,

$$
\begin{gathered}
E_{s}^{w}\left(\alpha_{N_{l}, s}^{w}\right)=2 \sum_{n=2}^{N_{l}} U_{n, s}^{w}\left(a_{n}\right) \leq 2 \sum_{n=2}^{N_{l}} U_{n, s}^{w}\left(x_{l}\right)=2 \sum_{n=2}^{N_{l}} \sum_{i=1}^{n-1} \frac{w\left(x_{l}, a_{i}\right)}{\left|x_{l}-a_{i}\right|^{s}} \\
\leq 2\|w\|\left[\frac{N_{l}-1}{\left|x_{l}-a_{1}\right|^{s}}+\frac{N_{l}-2}{\left|x_{l}-a_{2}\right|^{s}}+\cdots+\frac{1}{\left|x_{l}-a_{N_{l}-1}\right|^{s}}\right]=2\|w\|\left(S_{I, l}+T_{I, l}\right),
\end{gathered}
$$

where $\|w\|=\sup \{w(x, y): x, y \in[a, b]\}$ and

$$
S_{I, l}:=\sum_{a_{i} \in I, 1 \leq i \leq N_{l}-1} \frac{N_{l}-i}{\left|x_{l}-a_{i}\right|^{s}}, \quad T_{I, l}:=\sum_{a_{i} \notin I, 1 \leq i \leq N_{l}-1} \frac{N_{l}-i}{\left|x_{l}-a_{i}\right|^{s}} .
$$

For each $a_{i} \notin I,\left|a_{i}-x_{l}\right| \geq \epsilon / 2$; hence

$$
2 T_{I, l} \leq(2 / \epsilon)^{s} N_{l}^{2} .
$$

If $a_{i} \in I, 1 \leq i \leq N_{l}-1$, then $\left|a_{i}-x_{l}\right| \geq(d-c-\epsilon) / 2\left(\nu_{l}+1\right)$. Therefore, if we define $\tau_{l}:=\operatorname{card}\left\{1 \leq i \leq N_{l}-1: a_{i} \in I\right\}$, it follows that

$$
2 S_{I, l} \leq \frac{2^{s+1}}{(d-c-\epsilon)^{s}}\left(\nu_{l}+1\right)^{s} \tau_{l} N_{l} .
$$

By hypothesis, $\tau_{l}^{1+s} / N_{l}^{s} \rightarrow 0$ as $l \rightarrow \infty$. We deduce from (82)-(84) that

$$
\lim _{l \rightarrow \infty} \frac{E_{s}^{w}\left(\alpha_{N_{l}, s}^{w}\right)}{N_{l}^{1+s}}=0
$$

which contradicts the fact that

$$
\liminf _{N \rightarrow \infty} \frac{E_{s}^{w}\left(\alpha_{N, s}^{w}\right)}{N^{1+s}} \geq \lim _{N \rightarrow \infty} \frac{\mathcal{E}_{s}^{w}([a, b], N)}{N^{1+s}}=\frac{2 \zeta(s)}{\mathcal{H}_{1}^{s, w}([a, b])^{s}}>0
$$

\section{ACKNowledgments}

The authors wish to thank Professors A. Aptekarev and D. Hardin for many valuable discussions with us regarding the content of this paper. We are also grateful to N. Zorii for her careful reading of this manuscript and her suggestions which improved it. 


\section{REFERENCES}

[1] J. Baglama, D. Calvetti, and L. Riechel, Fast Leja points, Electron. Trans. Numer. Anal. 7 (1998), 124-140. MR1667643 (99j:65036)

[2] A.R. Bausch, M.J. Bowick, A. Cacciuto, A.D. Dinsmore, M.F. Hsu, D.R. Nelson, M.G. Nikolaides, A. Travesset, and D.A. Weitz, Grain boundary scars and spherical crystallography, Science 299 (2003), 1716-1718.

[3] J. Beck, The modulus of polynomials with zeros on the unit circle: A problem of Erdös, Ann. of Math. 134 (1991), 609-651. MR1135879 (93e:11091)

[4] S.V. Borodachov, D.P. Hardin, and E.B. Saff, Asymptotics for discrete weighted minimal Riesz energy problems on rectifiable sets, Trans. Amer. Math. Soc. 360 (2008), 1559-1580. MR 2357705 (2009a:49071)

[5] S.V. Borodachov, D.P. Hardin, and E.B. Saff, Asymptotics of best-packing on rectifiable sets, Proc. Amer. Math. Soc. 135 (2007), 2369-2380. MR2302558 (2008g:28027)

[6] J.S. Brauchart, D.P. Hardin, and E.B. Saff, The Riesz energy of the $N$-th roots of unity: an asymptotic expansion for large $N$, Bull. Lond. Math. Soc. 41 (2009), no. 4, 621-633. MR 2521357

[7] M. Bowick, D.R. Nelson, and A. Travesset, Interacting topological defects in frozen topographies, Phys. Rev. B62 (2000), 8738-8751.

[8] J.S. Brauchart, Optimal logarithmic energy points on the unit sphere, Math. Comp. $\mathbf{7 7}$ (2008), 1599-1613. MR2398782

[9] G. Choquet, Theory of capacities, Ann. Inst. Fourier 5 (1955), 131-295. MR0080760 $(18: 295 \mathrm{~g})$

[10] G. Choquet, Diamètre transfini et comparaison de diverses capacités, Technical report, Faculté des Sciences de Paris (1958).

[11] D. Coroian and P. Dragnev, Constrained Leja points and the numerical solution of the constrained energy problem, J. Comput. Appl. Math. 131 (2001), 427-444. MR1835725 (2002c:65241)

[12] S. De Marchi, On Leja sequences: some results and applications, Appl. Math. Comput. 152 (2004), 621-647. MR2062764 (2005c:41003)

[13] P.D. Dragnev and E.B. Saff, Riesz spherical potentials with external fields and minimal energy, Potential Anal. 26 (2007), 139-162. MR2276529 (2007k:31004)

[14] A. Edrei, Sur les déterminants récurrents et les singularités d'une fonction donnée par son développement de Taylor, Compositio Mathematica 7 (1939), 20-88. MR0001285(1:210c)

[15] T. Erber and G.M. Hockney, Complex systems: equilibrium configurations of $N$ equal charges on a sphere $2 \leq N \leq 112$, Advances in Chemical Physics, Vol. XCVIII, (1997), 495-594. MR1238214 (98e:78002)

[16] B. Farkas and B. Nagy, Transfinite diameter, Chebyshev constant and energy on locally compact spaces, Potential Anal. 28 (2008), 241-260. MR2386099(2009f:31003)

[17] B. Fuglede, On the theory of potentials in locally compact spaces, Acta Math. 103 (1960), 139-215. MR0117453 (22:8232)

[18] J. Górski, Les suites de points extrémaux liés aux ensembles dans l'espace à 3 dimensions, Ann. Math. Polon. 4 (1957), 14-20. MR0121770 (22:12504)

[19] M. Götz, On the distribution of Leja-Górski points, J. Comp. Anal. App. 3 (2001), 223-241. MR 1840565 (2002f:31016)

[20] D.P. Hardin and E.B. Saff, Minimal Riesz energy point configurations for rectifiable ddimensional manifolds, Adv. Math. 193 (2005), 174-204. MR2132763 (2005m:49006)

[21] A.B.J. Kuijlaars and E.B. Saff, Asymptotics for minimal discrete energy on the sphere, Trans. Amer. Math. Soc. 350 (1998), 523-538. MR1458327(98e:11092)

[22] N.S. Landkof, Foundations of Modern Potential Theory, Springer-Verlag, Heidelberg, 1972. MR0350027(50:2520)

[23] F. Leja, Sur certaines suites liée aux ensembles plans et leur application à la représentation conforme, Ann. Polon. Math. 4 (1957), 8-13. MR0100726 (20:7154)

[24] A. López García, Ph.D. Dissertation, Vanderbilt University.

[25] A. Martínez-Finkelshtein, V.V. Maymeskul, E.A. Rakhmanov, and E.B. Saff, Asymptotics for minimal discrete Riesz energy on curves in $\mathbb{R}^{d}$, Canad. J. Math. 56 (2004), 529-552. MR 2057285 (2005a:31010) 
[26] P. Mattila, Geometry of Sets and Measures in Euclidean Spaces, Cambridge University Press, Cambridge, 1995. MR 1333890 (96h:28006)

[27] M. Ohtsuka, On potentials in locally compact spaces, J. Sci. Hiroshima Univ. Ser. A-I 25 (1961), 135-352. MR0180695 (31:4926)

[28] E.B. Saff and A.B.J. Kuijlaars, Distributing many points on a sphere, Math. Intelligencer, 19 (1997), 5-11. MR1439152 (98h:70011)

[29] E.B. Saff and V. Totik, Logarithmic Potentials with External Fields, Grundlehren der Mathematischen Wissenschaften, Vol. 316, Springer-Verlag, 1997. MR1485778 (99h:31001)

[30] J. Siciak, Two criteria for the continuity of the equilibrium Riesz potentials, Comment. Math. Prace Mat. (1970), 91-99. MR0277742 (43:3475)

[31] N.V. Zorii, Equilibrium problems for potentials with external fields, Ukrainian Math. J. 55 (2003), 1588-1618. MR.2073873 (2005d:31010)

[32] N.V. Zorii, Equilibrium potentials with external fields, Ukrainian Math. J. 55 (2003), 14231444. MR2075132 (2005e:31010)

Center for Constructive Approximation, Department of Mathematics, Vanderbilt University, Nashville, Tennessee 37240

E-mail address: abey.lopez@vanderbilt.edu

Center for Constructive Approximation, Department of Mathematics, Vanderbilt University, Nashville, Tennessee 37240

E-mail address: edward.b.saff@vanderbilt.edu 